\title{
Nature and magnitude of operating forces in a horizontal bend conveying gas-liquid slug flows
}

\author{
Mustapha Gourma ${ }^{\mathrm{a}, *}$, Patrick G. Verdin ${ }^{\mathrm{b}}$ \\ ${ }^{a}$ Cranfield University, Energy \& Power Engineering Institute. Cranfield MK43 0AL UK \\ ${ }^{b}$ Cranfield University, Thermal Energy Systems and Materials. Cranfield MK43 0AL UK
}

\begin{abstract}
Operating forces and magnitude of loads from gas-liquid slug flows exerted on a horizontally orientated $90^{\circ}$ bend are investigated. The distributed forces are either Newtonian, associated with the fluids motion or Configurational, inherent to the internal distributions of the phases. The forces are derived through the conventional balances of mass and linear momentum arising from the volume of fluid (VOF) description of gas-liquid flows. The study uses the integral form of the momentum balance to estimate the operating forces budget. Invoking dynamical time scales separation discloses the connection of the Lamb vector (vortex-force) to the local time rate of momentum. An interesting outcome being an explicit expression for Favre-Reynolds stress that reveals the contribution of void fraction fluctuations in the redistribution of the stress across the interface.

Numerical simulations are performed to determine the magnitude of Newtonian loads on bend using a segmented domain technique to represent the fully established slug flow regime. The time-dependent traces of the relevant flow variables such as liquid hold-up, flow rates and resultant forces on the bend are recorded and analysed. Compared to the isotropic component, the deviatoric stresses are shown to have a marginal contribution to the total forces.
\end{abstract}

\footnotetext{
*Corresponding author.Tel:+44(0) 1234 755035, Fax:+44(0)1234 754746

Email address: gourma.m@gmail.com (Mustapha Gourma )
} 
It is also shown that loading cycles on bends are much higher than slugging cycles; this is an important feature for the structural integrity assessment of pipelines with bends.

Keywords: Slug flow, Slug impact, Singular interface, Lamb vector, Favre-Reynolds stress 


\section{Nomenclature}

$C_{2 \epsilon}^{*} \quad$ Empirical parameter

$C_{\sigma}$ Contact line of two fluids

$C_{1 \epsilon} \approx 1.42$ Empirical parameter

$C_{3 \epsilon} \approx 1.68$ Empirical parameter

$C_{\mu} \approx 0.0845$ Experimental constant

$D$ pipe diameter, $m$

$F$ Surface tension, $N / m$

$G_{b}$ Buoyancy term for turbulent kinetic energy generation

$G_{k}$ Resolved term for turbulent kinetic energy generation

$I_{d}$ Identity tensor

$Q_{k}$ Phasic mass flow rate, $\mathrm{kg} / \mathrm{s}$

$S$ Entropy, $J K^{-1}$

$S_{k}$ Phasic entropy, $J K^{-1}$

$S_{V_{k}}=\frac{p V_{k}}{T}$ Pseudo entropy, $J K^{-1}$

$T$ Temperature, $K$

$V$ Mixture specific volume, $m^{3}$

$V_{k}$ Phasic specific volume, $m^{3}$

$\hat{n}$ Unit normal to the interface

$\mathcal{R}_{\mathcal{F}}^{k}$ Phasic Favre-Reynolds stress, $\mathrm{m}^{2} / \mathrm{s}^{2}$

$\mathcal{R}_{\mathcal{F}}$ Favre-Reynolds stress, $\mathrm{kg} \mathrm{m} / \mathrm{s}^{2}$

$\mathcal{R}_{c}$ Compressible Reynolds stress, $\mathrm{kg} \mathrm{m} / \mathrm{s}^{2}$

$\mathcal{R}_{s}$ Standard Reynolds stress, $\mathrm{m}^{2} / \mathrm{s}^{2}$

$\mathcal{T}$ The total stress, $\mathrm{Pa}-\mathrm{s}$

$\bar{u}$ Reynolds mean velocity, $\mathrm{m} / \mathrm{s}$

$\tilde{u}$ Favre mean velocity, $\mathrm{m} / \mathrm{s}$

$c_{k}$ Isothermal phasic speed of sound, $\mathrm{m} / \mathrm{s}$

$g$ acceleration due to gravity, $\mathrm{m} / \mathrm{s}^{2}$

$n_{\sigma}$ Unit normal to singular interface

$n_{C_{\sigma}}$ Unit normal to contact line

$n_{\partial \sigma}$ Unit normal to contact line and wall

$p$ pressure, $\mathrm{Pa}$

$p_{k}$ Phasic pressure, $\mathrm{Pa}$

$t_{C_{\sigma}}$ Unit tangent to contact line

$t_{\partial \sigma}$ Unit tangent to contact line and wall

$u$ mixture velocity, $\mathrm{m} / \mathrm{s}$

$u^{\prime \prime}$ Favre fluctuating velocity, $\mathrm{m} / \mathrm{s}$

$u_{k}^{\prime}$ Reynolds fluctuating velocity, $\mathrm{m} / \mathrm{s}$

$u_{T S}$ Translational slug velocity, $\mathrm{m} / \mathrm{s}$

\section{Greek symbols}

$\alpha_{k}$ Phasic volume fraction

$\beta$ Thermal expansion coefficient, $K^{-1}$ $\beta_{\epsilon} \approx 0.012$ Empirical constant

$\chi$ A regular function

$\delta_{\sigma}$ Dirac measure over the interface $\sigma(t)$

$\epsilon$ Turbulent dissipation, $\mathrm{m}^{2} / \mathrm{s}^{2}$

$\eta$ Bulk viscosity, $\mathrm{Pa}-s$

$\Gamma_{k}$ Phasic Grüneisen parameter

$\gamma_{k}$ Ratio of specific heat coefficient

$\gamma_{i j}$ Surface tension per mixture density $i$ and $j, \mathrm{~m}^{3} / \mathrm{kg}$

$\lambda$ Dynamic viscosity, $\mathrm{Pa}-s$

$\mathcal{K}$ Turbulent kinetic energy, $\mathrm{m}^{2} / \mathrm{s}^{2}$

$\mu$ Dynamic viscosity, $\mathrm{Pa}-\mathrm{s}$

$\mu_{t}$ Turbulent viscosity, $\mathrm{Pa}-\mathrm{s}$

$\Omega$ Domain of control volume, $\mathrm{m}^{3}$

$\Omega_{k}$ Phasic control volume, $m^{3}$

$\omega_{k}$ Phasic vorticity, $s^{-1}$

$\bar{\psi}$ Reynolds mean value of a field

$\partial \Omega$ Boundary of the domain, $m^{2}$

$\psi$ Instantaneous field

$\psi^{\prime}$ Fluctuation of instantaneous field

$\rho$ Mixture density, $\mathrm{kg} / \mathrm{m}^{3}$

$\rho_{k}$ Phasic density, $\mathrm{kg} / \mathrm{m}^{3}$

$\sigma(t)$ Singular interface: time dependent

$\sigma_{\epsilon}$ Prandtl turbulent number

$\sigma_{\mathcal{K}}$ Prandtl turbulent number

$\sigma_{i j}$ Surface tension coefficient

$\tau$ Shear stress, $\mathrm{Pa}$

$\tau_{p}$ Time scale of pressure, $s$

$\tau_{S}$ Time scale of entropy, $s$

$\tau_{\rho u}$ Dynamical time scale of mass flow, $s$

$\tau_{\rho}$ Dynamical time scale of density, $s$

$\tau_{u}$ Dynamical time scale of velocity, $s$

$\tilde{\psi}$ Favre mean value of a field

$\varkappa_{i}$ Curvature from phase i, $m^{-1}$

$\xi_{0} \approx 4.38$ Empirical constant

$\xi_{\epsilon}$ Ratio of turbulent to mean strain time

$\xi_{k}$ Phasic mass fraction

$d \delta_{C_{\sigma}}$ Lebesgue measure of contact line $C_{\sigma}$ Subscripts

$\sigma$ interface

$C$ contact line

$g$ gas

$k$ phase

$l$ liquid 


\section{Introduction}

Two-phase flows are widely encountered in many processes involving chemical systems, nuclear plants and hydrocarbons transportations. Among the variety of two phase flow regimes occurring in industrial and engineering applications, the slug flow regime is of particular interest because of its unstable and intermittent nature. The analyses of the slug flow regime are approachable through statistical perspectives only since all the flow characteristics, such as the slug frequency, the slug length and the slug translational velocity are subject to local fluctuations.

Fluctuating forces and flow-induced vibrations resulting from slug flows are of concern in industrial facilities for safety and production integrity. Bends and tees are often present in industrial flow lines and are usually unavoidable in confined spaces such as laboratories. The primary mechanical design is critical for prolonging the life time of such fittings and for preventing their damage and failure. Identifying natural frequencies of flow lines is also necessary to avoid resonance with slug flow mean frequencies.

Five decades ago, researchers pioneered investigations on vapour-water two phase flow interactions in vertical structures $[1,2]$. They outlined the importance of the unsteadiness of the momentum fluxes in exciting appreciable structural vibrations. More recently, slug flow-induced forces on horizontal bends were investigated in a series of experiments $[3,4]$, where forces due to slugs passage in a bend were measured; a correlation was established between magnitude of loads and slug dynamics. Their analysis also showed the negligible effects of surface tension and liquid viscosity on these loads at least at low pressure. In a recent investigation the same authors validated a 1D Piston Flow Model PFM with their own data via the traces of transient maximum forces and pressures [5]. A STAROLGA coupling was performed using the 3-D CFD code StarCCM+ and the 1-D code OLGA in a horizontally oriented $90^{\circ}$ bend [6]. Results showed reasonable 
agreement of force magnitude on the bend with the data reported in [3]. A study with OpenFoam by [7] predicted instantaneous component of forces on a bend that were well within the range of the experimental data. The shape of the power spectral density $P S D$ of the forces was also in agreement with measurements. Although $R A N S k-\omega S S T$ and $L E S$ simulations nicely predicted liquid hold-up distributions, the root mean squared RMS of forces were $30 \%$ under-valued by both methods. Moreover, LES under-predicted the average and standard deviation of the hold-up compared to RANS $k-\omega S S T$.

Measurements of transient forces in bubbly and churn air-water flow regimes in a vertically orientated elbow and tee were reported in [8]. After assessing the effects of various are in flow parameters, the authors extended the correlation of the maximum RMS (root mean square) forces to the Weber number (measure of the relative importance of the fluid's inertia compared to its surface tension) provided in [2]. Experiments in a two-phase vertical tube bundle carried out in [9] showed that the quasi-periodic drag forces and the quasi-periodic lift forces are correlated to the fluctuations of the momentum balance and to the oscillations in the wake of the cylinders, respectively. The authors developed predictive semi-analytical models correlating their findings. Similarly, the authors in [10] conducted experiments in a vertical system, covering bubbly, slug, churn and annular flow regimes. After shunting the frequency of the flow induced vibrations, the authors were able to analyse the fluctuating hydrodynamical variables purely inherent to the flow mixture. With an elaborated semi-analytical formulation based on a two phase mixture model, they derived the acting force spectrum on the elbow through a wave form (Fourier transform). A sudden jump was observed in the force fluctuation at a given liquid flow rate during the transition from a bubbly to a slug flow regime. It was also found that the elbow damped the high frequency terms of momentum flux fluctuations. Experiments of gas-liquid stratified wavy flows 
in a horizontal test section are described in [11]. The authors were able to take into account the collisional effects resulting from gas-liquid waves and structure interaction and they established that wave collision forces and momentum fluctuations play a major role in the considered regime. Their predictions of both the peak force fluctuations and the peak frequency were $25 \%$ accurate. This work is directed towards investigations of air-water slugs in a horizontal bend using the volume of fluid (VOF) approach, where fluid components are separated and are in pressure-temperature equilibrium with a shared velocity. The first part of the study focuses on deriving theoretical estimates of forces from the mass and momentum balances arising from the VOF formulation. The derivation of configurational forces is carried out under the assumption of separated flows in the framework of smooth singular interfaces which is justified for slug and stratified flow regimes.

The term "singular interface", also known as "material interface" is regularly mentioned here. It is important to note that in singular interfaces, exchanges of mass between adjacent phases are not permitted, whereas fluid elements can cross non-material interfaces.

Regarding the estimate of the local time rate momentum, the authors in [10] made the use of Fourier transform to derive the force spectrum. The current approach considers that the local time rate momentum operates on distinguishable dynamical time scales involving pressure waves with fast time scale, and material waves on slower time scale. Exploiting the separation of slow and fast time scales permits to ignore the slow time scale component contribution in the local time rate momentum. To the authors' best knowledge, such approximation has never been reported in the literature. Regardless of its restriction, it highlights the connection of the Lamb vector "vortex-force" with the time rate change of the momentum as well as with the contact line force. 
For the evaluation of the convective momentum, Reynolds' decomposition is invoked for the mixture velocity and the contribution of void fraction fluctuations is highlighted. Moreover, a useful expression of Favre-Reynolds stress is obtained using Grüss-type-inequality. This expression sheds light on the compressibility effect of the fluctuating stress across the interface, which is related to turbulence redistribution at interfaces, a central phenomenon in fluid dynamics. Detailed investigations on the subject can be found in [12-16] for instance.

Contrary to the flow in vertical lines, numerical studies in horizontal lines are highly sensitive to the upstream conditions. In order to eliminate the upstream effects and to reproduce a fully developed slug flow regime in relatively short lines, a segmented domain technique (SDT) is implemented where transient flow properties obtained at the outlet section of a long separate pipe section are introduced at the inlet of the domain to be studied, thus limiting the required entrance length and reducing the overall computational time, see [17] for a detailed description of this approach.

The second aim of the present work is to estimate the resultant of flow forces on the bend surface via numerical simulations. To that end, flow rates, volume fractions, magnitude of pressures and total forces are analysed. It is observed that at low flow rates, a liquid film travelling into the bend experiences a crosssectional acceleration when centrifugal forces prevail, inducing a "film inversion" phenomenon. A correlation for the inception of such phenomenon has been established in [18].

\section{Mathematical model}

The standard volume of fluid (VOF) model, originated in [19], is adopted in this work on slug flows. As the slug flow regime is commonly characterised by its turbulent gas phase, the VOF model is used in combination with a turbulence model. The RNG $k-\epsilon$ model has been selected, this choice being motivated by 
the work presented in [20].

\subsection{Volume of Fluid model}

The VOF model is available in most commercial CFD software. This model is based on the assumption that two or more fluids are not inter-penetrating. Variables and properties in each cell are functions of the phase fractions, as detailed in the ANSYS Fluent user's guide [21]. Based on this definition, the continuity equations for liquid and gas volume. fractions, $\alpha_{l}$ and $\alpha_{g}$, can be written as:

$$
\partial_{t} \rho_{k} \alpha_{k}+\nabla \cdot\left(\rho_{k} \alpha_{k} \vec{u}\right)=0
$$

where the subscript $k=g, l$ denotes either the liquid or the gas phase. The momentum equation uses a single velocity $\vec{u}$ acting on the mixture with a density $\rho=\alpha_{g} \cdot \rho_{g}+\alpha_{l} \cdot \rho_{l}$ and a viscosity $\mu=\alpha_{G} \cdot \mu_{g}+\alpha_{l} \cdot \mu_{l}$. The momentum balance in conservative form is:

$$
\partial_{t} \rho \vec{u}+\nabla \cdot(\rho \vec{u} \otimes \vec{u})=\nabla \cdot \mathcal{T}+\rho \cdot \vec{g}+\vec{F}
$$

The symbol $\otimes$ in Equation 2 stands for the dyadic product, $\mathcal{T}=\mathcal{T}(p, \tau)$ is a tensor which contains the pressure $p$ and the viscous stress tensor $\tau$ given by:

$$
\mathcal{T}=-p \cdot I_{d}+\tau
$$

with $I_{d}$ is the identity matrix and the shear viscous stress tensor is expressed by $\tau=\mu \cdot\left(\nabla \vec{u}+\nabla \vec{u}^{T}\right)+\left(\eta-\frac{2}{3} \mu\right)(\nabla \cdot \vec{u}) \cdot I_{d}$ also denoted $\tau(\mu, \eta, \vec{u})$.

The coefficients $\mu$ and $\lambda=\left(\eta-\frac{2}{3} \mu\right)$ are the dynamic viscosity and the bulk viscosity of the mixture, respectively.

For Newtonian fluids, the divergence term in $\tau$ is usually important in regions under shocks (compression/expansion). For fluids in confined systems, the shear is larger and dominant due to the wall boundaries. The last term on the right hand 
side of Equation 2 represents the inter-facial surface tension $F$ between phases $i$ and $j$ which is given by:

$$
\vec{F}=\sigma_{i j} \cdot \frac{\rho \cdot \varkappa_{i} \cdot \vec{\nabla} \alpha_{i}}{\frac{1}{2}\left(\rho_{i}+\rho_{j}\right)}
$$

The curvature $\varkappa_{i}$ can be expressed in terms of the gradient of the volume fraction $\vec{\nabla} \alpha_{i}=\overrightarrow{n_{i}}$ as:

$$
\varkappa_{i} \cdot \vec{\nabla} \alpha_{i}=-\left(\nabla \cdot \frac{\vec{\nabla} \alpha_{i}}{\left\|\vec{\nabla} \alpha_{i}\right\|}\right) \cdot \overrightarrow{n_{i}}=-\left(\nabla \cdot \hat{n}_{i}\right) \cdot \overrightarrow{n_{i}}
$$

Indices $i$ and $j$ represent again the two phases. The surface tension coefficient $\sigma_{i j}$ is assumed constant, and $\hat{n}_{i}=\frac{\overrightarrow{n_{i}}}{\left\|\overrightarrow{n_{i}}\right\|}$ is the unit normal to the interface where the surface tension is calculated. The expression of the inter-facial force $\vec{F}$ becomes:

$$
\begin{aligned}
\vec{F} & =-\frac{\sigma_{i j}}{\frac{1}{2}\left(\rho_{i}+\rho_{j}\right)} \cdot \rho\left(\nabla \cdot \hat{n}_{i}\right) \cdot \vec{n}_{i} \\
& =-\gamma_{i j} \cdot\left(\nabla \cdot \hat{n}_{i}\right) \cdot \vec{n}_{i}
\end{aligned}
$$

\section{2. $R N G k-\epsilon$ turbulence model}

The $R N G k-\epsilon$ turbulence scheme belongs to the two-equation eddy-viscosity turbulence model family. It has been derived from the re-normalisation group theory [22] with scale expansion for Reynolds stress. It has been a standard in engineering applications for some time now due to its good balance between numerical demands and stability. The scheme is semi-empirical in its approach. It is common to decompose instantaneous variables $\vec{\psi}$ following Favre or Reynolds' decomposition, into $\vec{\psi}=\bar{\psi}+\vec{\psi}^{\prime}$. Where the averaged term $\bar{\psi}$ representing large scales and fluctuating small scales term $\vec{\psi}^{\prime}$. Hence two additional transport equations need to be solved to compute the Reynolds stresses. The first transport equation is for the turbulent kinetic energy $k=u^{\prime} \otimes u^{\prime} / 2$, and the second transport equation is for the rate of turbulence dissipation $\epsilon$ :

$$
\partial_{t} \rho \cdot k+\nabla \cdot(\rho \bar{u} k)=\nabla \cdot\left(\left(\mu+\frac{\mu_{t}}{\sigma_{k}}\right) \nabla k\right)+G_{k}+G_{b}-\rho \cdot \epsilon
$$




$$
\partial_{t} \rho \cdot \epsilon+\nabla \cdot(\rho \bar{u} \epsilon)=\nabla \cdot\left(\left(\mu+\frac{\mu_{t}}{\sigma_{\epsilon}}\right) \nabla \epsilon\right)+C_{1 \epsilon} \frac{\epsilon}{k}\left(G_{k}+C_{3 \epsilon} G_{b}\right)-C_{2 \epsilon}^{*} \cdot \rho \frac{\epsilon^{2}}{k}
$$

The source term of turbulence $G_{k}$ appearing in Equations 7 and 8 stands for the generation of turbulent kinetic energy due to the resolved velocity gradients and $G_{b}$ is the generation of the turbulent energy due to buoyancy:

$$
\left\{\begin{array}{l}
G_{b}=-g \frac{\mu_{t}}{\rho \cdot \operatorname{Pr}} \nabla \rho \\
G_{k}=\mu_{t} \cdot\left(\nabla \bar{u}+\nabla \bar{u}^{T}\right) \cdot \nabla \bar{u}^{T}-\frac{2}{3} k \cdot \nabla \bar{u}
\end{array}\right.
$$

The turbulent viscosity $\mu_{t}$ is derived from $k$ and $\epsilon$ and involves an experimental constant $C_{\mu} \simeq 0.0845[21]$ :

$$
\mu_{t}=\rho \cdot C_{\mu} \cdot \frac{k^{2}}{\epsilon}
$$

Quantities $C_{1 \epsilon}=1.42, C_{3 \epsilon}=1.68$ are empirical constants [21] while $\sigma_{\epsilon}$ and $\sigma_{k}$ are turbulent Prandtl numbers. In Equation $8, C_{2 \epsilon}^{*}$ is function of the scale expansions for the Reynolds stress $\xi_{\epsilon}$ as follows:

$$
\left\{\begin{array}{l}
C_{2 \epsilon}^{*}=1.92+\frac{C_{\mu} \cdot \xi\left(1-\xi_{\epsilon} / \xi_{0}\right)}{1+\beta_{\epsilon} \xi_{\epsilon}^{3}} \rho \xi_{\epsilon}^{3} \\
\xi_{\epsilon}=\mu_{t} \cdot \sqrt{\tau \otimes \tau} \cdot \frac{k}{\epsilon}
\end{array}\right.
$$

where $\tau=\left(\nabla \bar{u}+\nabla \bar{u}^{T}\right)$ is the resolved strain. The parameter $\xi_{\epsilon}$ gives the ratio of turbulent to mean strain time scales, and the default constant are $\beta_{\epsilon}=0.012$ and $\xi_{0}=4.38[21]$.

Transport equations are solved for $k$ and $\epsilon$. The turbulent viscosity $\mu_{t}$ is computed and the Reynolds stresses are determined and substituted into the momentum equations. The new velocity components are used to update the turbulence generation term $G_{k}$, and the process is repeated. 


\section{Momentum flux}

The mixture flow rate distributions and fluctuations in the elbow are directly accountable for the forces induced on the bend wall. The analysis of these forces can be better understood when not looking at the instantaneous local balances in the governing relations (Equations 1 and 2) but rather at their integral counterparts over a specific control volume $\Omega$ of the bend, as illustrated in Figure 1. In the following, the force due to gravity will be omitted from the derivation.

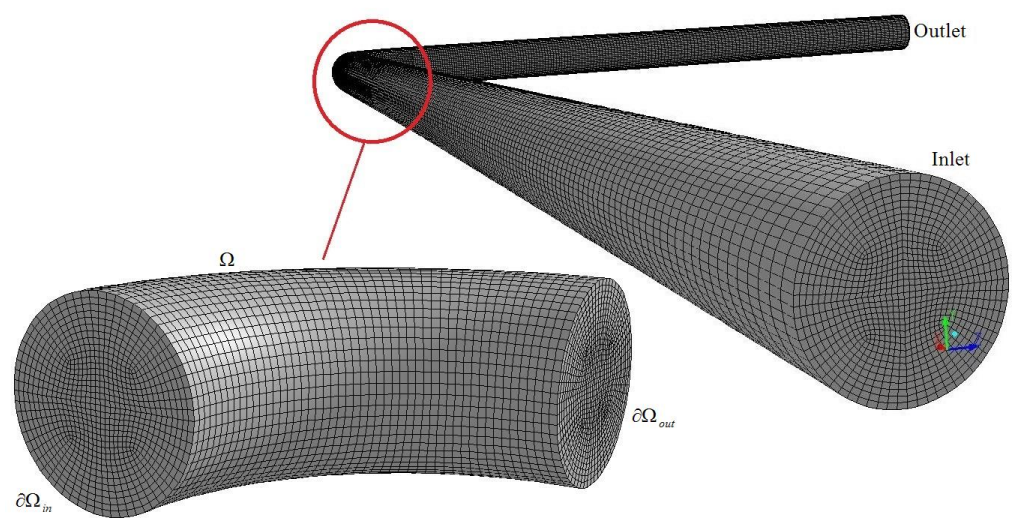

Figure 1: Schematic representation of the control volume $\Omega$ of $90^{\circ}$ bend

Slug flow is considered in a fixed control volume $\Omega$ delimited by its surrounding control surface $\partial \Omega=\partial \Omega_{\text {in }} \cup \partial \Omega_{L} \cup \partial \Omega_{\text {out }}$, with $\partial \Omega_{\text {in }}, \partial \Omega_{L}$ and $\partial \Omega_{\text {out }}$ the inlet, lateral and outlet surfaces, respectively. Taking into account Reynolds' decomposition for the convective term, the resulting rate of change of momentum in a fixed control volume becomes:

$$
\begin{aligned}
\partial_{t} \int_{\Omega} \rho \cdot u d \Omega+\int_{\Omega} \nabla \cdot[\rho u \otimes u] d \Omega & =\int_{\Omega} \nabla \cdot\left[-p I_{d}+\tau\right] d \Omega \\
& +\int_{\Omega} \rho \cdot \vec{g} d \Omega \\
& -\int_{\Omega} \gamma_{l g} \cdot\left(\nabla \cdot \hat{n}_{i}\right) \vec{n}_{i} d \Omega
\end{aligned}
$$




\subsection{Contact line momentum on bend}

The last term on the right hand side of Equation 12 represents the momentum exchange between the interface and the control volume. The time dependent interface between the gas and the liquid is represented by $\sigma(t)=\Omega_{g}(t) \cap \Omega_{l}(t)$ whereas $C_{\sigma}(t)=\partial \Omega \cap \sigma(t)$ represents the contact line of the two fluids with the bend wall, see Figure 2.
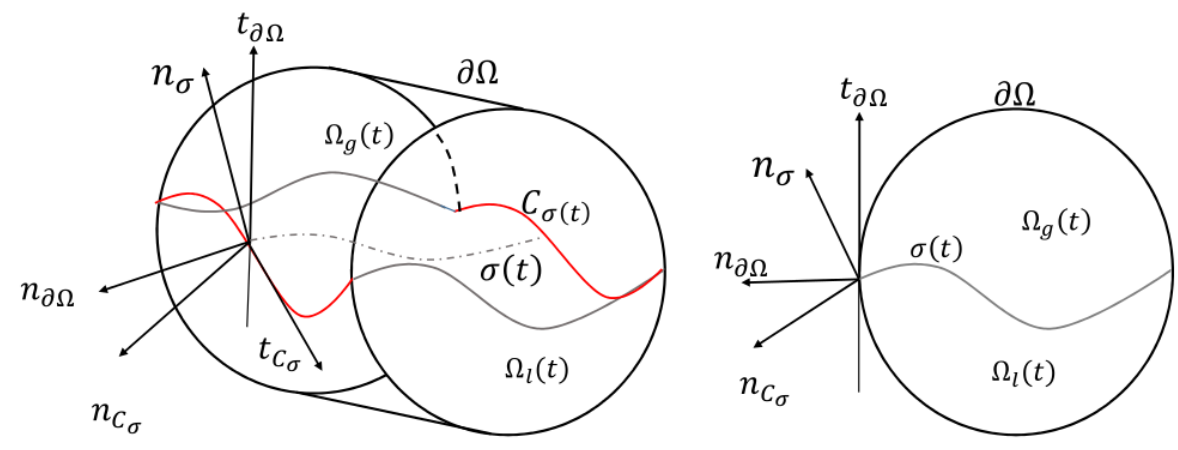

Figure 2: Representation of the domains and various unit vectors, in perspective (left) and in orthogonal plane to $t_{\partial \Omega}$ (right)

Two sets $\left(t_{C_{\sigma}}, n_{\sigma}, n_{C_{\sigma}}\right)$ and $\left(t_{C_{\sigma}}, t_{\partial \Omega}, n_{\partial \Omega}\right)$ are defined in Figure 2 , which are positively orientated orthonormal basis. The unit vectors defined on the boundaries and at the interface $\sigma(t)$ are defined as follows: $t_{C_{\sigma}}=n_{\sigma} \wedge n_{\partial \Omega}$ and $n_{C_{\sigma}}=t_{C_{\sigma}} \wedge n_{\sigma}$ are the tangent and normal vectors to the contact line $C_{\sigma}(t)$, and $t_{\partial \Omega}=n_{\partial \Omega} \wedge t_{C_{\sigma}}$ is the tangent vector to the boundary.

If the distribution $\delta_{\sigma}$ represents the "Dirac measure" of the interface $\sigma(t)$, then its eventual "Lebesgue measure" $d \sigma(s)=d \delta_{\sigma}(s)$ counter part, or Lebesgue integral for any regular field $\chi$, satisfies:

$$
\left\langle\delta_{\sigma}, \chi\right\rangle=\int_{\Omega} \chi(s) \cdot d \sigma(s)=\chi(\sigma)
$$

Similarly for the contact line $C_{\sigma}(t)$, the "Lebesgue measure", $d C_{\sigma}(s)=d \delta_{C_{\sigma}}(s)$ 
satisfies the following Lebesgue integral:

$$
\left\langle\delta_{C_{\sigma}}, \chi\right\rangle=\int_{\partial \Omega \cap \sigma} \chi(s) \cdot d C_{\sigma}(s)=\chi\left(C_{\sigma}\right)
$$

where $s$ stands for a material point on the interface where the mass of the measure is concentrated. The momentum exchange due to the interface in Equation 12 can be reformulated as:

$$
\int_{\Omega} \gamma_{l g}\left(\nabla \cdot \hat{n}_{i}\right) \vec{n}_{i} d \Omega=\int_{\sigma(t)} \sigma_{l g}\left(\nabla \cdot \hat{n}_{\sigma}\right) \vec{n}_{\sigma} d \sigma
$$

Following the surface curvature-divergence relation, see [23], the right hand side of Equation 15 can be decomposed as:

$$
\begin{aligned}
\int_{\sigma(t)} \sigma_{l g}\left(\nabla \cdot \hat{n}_{\sigma}\right) \vec{n}_{\sigma} d \sigma & =\int_{C_{\sigma}(t)} \sigma_{l g} \cdot \vec{n}_{C_{\sigma}} d C_{\sigma} \\
& -\int_{\sigma(t)} \nabla_{\sigma}\left[\sigma_{l g}\right] d \sigma
\end{aligned}
$$

The first term in Equation 16 refers to the contact force of the interface boundary (contact line gas-liquid-wall) with the bend. The second term is a tangential force, a purely internal force to $\sigma(t)$, resulting from the non-homogeneous interface density and has no effect on the bend wall. The surface gradient $\nabla_{\sigma}=\left(I-\hat{n}_{\sigma} \otimes\right.$ $\left.\hat{n}_{\sigma}\right) \cdot \nabla$ in the last term of Equation 16 is the orthogonal projection of the total gradient $\nabla$ onto the tangential space to $\sigma(t)$.

A constant mixture density along the contact line as well as at the interface is assumed, $\rho(\sigma) \approx \frac{1}{2}\left(\rho_{g}+\rho_{l}\right)=\rho\left(C_{\sigma}\right)$; it follows that the tangential force vanishes.

The forces in Equation 16 can be evaluated for a smooth and regular contact line as in the case of a film inversion. When the slug body impacts the elbow, the contact lines become complex and non-measurable.

\subsection{Pressure and mean stress momentum on bend}

To carry out the estimation of forces in the control volume in Equation 12, the flows of the two phases are considered continuously distributed into regular 
subsets $\Omega_{k}(s, t)$ with $k=g, l$, denoting again the gas and liquid phases, respectively. These flows are partitioned by an oriented moving singular interface $\sigma(s, t)$. The sub-regions $\Omega_{k}(s, t)$ are proper subsets contained in the entire domain $\Omega$, i.e. $\Omega \supseteq \Omega_{g} \cup \Omega_{l}$; and $\Omega_{g} \cap \Omega_{l}=\sigma$ as described in Figure 2. It should be noted that $\Omega_{k}(s, t)$ are non material volumes (in a Lagrangian sense). The hypothesis of continuous phase distributions implies that any mixture variable $\psi(s, t)$ obeys: $\lim _{s \rightarrow \Omega_{k}} \psi(s, t)=\psi_{k}$. Under these considerations and on the basis of the extended Green-Gauss theorem, the stress momentum becomes (see Appendix A):

$$
\begin{aligned}
\int_{\Omega_{l} \cup \Omega_{g}} \nabla \cdot\left[-p I_{d}+\tau\right] d \Omega & =\sum_{j=\text { in }}^{\text {out }} \sum_{k=g, l} \int_{\partial \Omega_{j}}\left(-p_{j} I_{d}+\tau_{k}\right) d \vec{S}_{j} \\
& +\int_{\partial \Omega_{L}}\left(-p_{L} I_{d}+\tau_{L}\right) d \vec{S}_{L} \\
& +\int_{\sigma}\left[\left[-p I_{d}+\tau\right]\right] \hat{n}_{\sigma} d \sigma
\end{aligned}
$$

The term $[[\psi]]=\psi_{l}-\psi_{g}$ stands for the generic finite jump of the quantity $\psi$ across the interface $\sigma$. The first term on the right hand side of Equation 17 represents the forces applied on the inlet and outlet flows, the second term stands for the operating load of the flow on the bend, and the last term stands for the internal force resulting from the jump in the stress across the interface.

\subsection{Local time rate of linear momentum on bend}

The time scale $\tau_{\rho u}$ over which the local time rate of momentum $\partial_{t} \rho u$ evolves, arises from interactions of processes of mixture density and velocity evolutions. To approximate the local momentum, the actual approach is based on time scales separation between $\tau_{u}$ of the mixture velocity and $\tau_{\rho}$ of the mixture density (void fractions). The time scale $\tau_{u}$ is related to the slow dynamics of material wave while $\tau_{\rho}$ is associated with characteristic times of pressure $p$ and thermal $T$ (entropy $S)$ waves. This association follows the connection of continuity equation along with Gibbs free energy of a mixture in $\mathrm{p}-\mathrm{T}$ pressure-temperature equilibrium, 
as indicated in Equation 18 for the individual phases $k=g, l$ (see Appendix D, equation D.9 for details).

$$
\frac{1}{p} \partial_{t} p=\frac{\gamma_{k}}{\rho_{k}} \partial_{t} \rho_{k}+\frac{\Gamma_{k}}{S_{V_{k}}} \partial_{t} S_{k}
$$

where $\gamma_{k}$ and $\Gamma_{k}$ stand for the adiabatic exponent and the Grüneisen parameter, respectively. The quantity $S_{V_{k}}=\frac{p V_{k}}{T}$ is the entropy analogue, which is also homogeneous to a heat capacity coefficient. A direct observation of Equation 18 shows that both entropy and density time rates are driven by pressure time rate.

The following dynamical time-scales are considered, $\tau_{\psi}=\left(\partial_{t} \psi / \psi\right)^{-1}$, where $\psi=\rho_{k}$ and $p$. The expression $\tau_{S_{k}}=\left(\partial_{t} S_{k} / S_{V_{k}}\right)^{-1}$ is their approximate counterpart for the entropy $S_{k}$. Equation 18 thus becomes:

$$
\begin{aligned}
\frac{1}{\tau_{p}} & =\frac{\gamma_{k}}{\tau_{\rho_{k}}}+\frac{\Gamma_{k}}{\tau_{S_{k}}} \\
\frac{1}{\tau_{p}} & =\frac{1}{\tau_{\rho_{k}}}+\Gamma_{k}\left(\frac{\beta T}{\tau_{\rho_{k}}}+\frac{1}{\tau_{S_{k}}}\right)
\end{aligned}
$$

where $\gamma_{k}=1+\Gamma_{k} \beta T$. According to Menikoff and Plohr (1989) in [24] thermodynamic consistency imposes the positiveness of the thermal expansion $\beta>0$ away from the phase transition. In addition, the existence of stable shock waves requires $\Gamma_{k}>0$, hence $\gamma>1$. Consequently $\tau_{p}<\tau_{\rho_{k}}$, implying that phasic density disturbances undergo dynamic changes slower than pressure changes. Regarding the entropy disturbances, a similar reasoning holds only for $\Gamma_{k} \geq 1$.

The derivation of continuity and momentum equations for compressible turbulent flow are recalled in Appendix C. The integral of instantaneous local time rate of momentum expressed in terms of dynamical time scales states:

$$
\begin{aligned}
\partial_{t} \int_{\Omega} \bar{\rho} \tilde{u} d \Omega & =\int_{\Omega} \bar{\rho} \tilde{u}\left(\partial_{t}(\bar{\rho} \tilde{u}) / \bar{\rho} \tilde{u}\right) d \Omega \\
& =\int_{\Omega} \bar{\rho} \tilde{u} / \tau_{\rho u} d \Omega \\
& =\int_{\Omega} \bar{\rho} \tilde{u}\left(1 / \tau_{\rho}+1 / \tau_{u}\right) d \Omega
\end{aligned}
$$


where, $\tau_{\rho}$ and $\tau_{u}$ refer to dynamical time scale inherent to mixture density $\rho$ and mixture velocity $u$. In subsonic systems, $\tau_{\rho} \ll \tau_{u}$, hence only the short time scale (fast dynamics) contribution is preserved in the evaluation of the local time rate of acceleration.

$$
\partial_{t} \int_{\Omega} \bar{\rho} \cdot \tilde{u} d \Omega \simeq \int_{\Omega} \tilde{u} \partial_{t} \bar{\rho} d \Omega
$$

Note that the assumption outlined in the previous section (smoothly separated phases), the local momentum approximation becomes (see Appendix B):

$$
\begin{aligned}
\int_{\Omega_{g} \cup \Omega_{l}} \tilde{u} \partial_{t} \bar{\rho} d \Omega & =-\sum_{k=g, l} \int_{\Omega_{k}} \nabla \cdot \rho_{k} \bar{\alpha}_{k}\left(\tilde{u}_{k} \otimes \tilde{u}_{k}-\frac{\left|\tilde{u}_{k}\right|^{2}}{2} I_{d}\right) d \Omega_{k} \\
& -\int_{\sigma} \frac{\left|\tilde{u}_{\sigma}\right|^{2}}{2} I_{d}[[\rho \bar{\alpha}]] \hat{n}_{\sigma} d \sigma+\sum_{k=g, l} \int_{\Omega_{k}} \rho_{k} \bar{\alpha}_{k} \omega_{k} \wedge \tilde{u}_{k} d \Omega_{k}
\end{aligned}
$$

The volumetric terms appearing in the Equation 22 are forces resulting from the interplay between the conservative and the non-conservative convective forces as is the interfacial force that is due to jump in kinetic energy across the interface. The last term is the vorticity-velocity cross product term known as the Lamb vector, also termed "vortex force", often denoted $\mathcal{L}_{B_{k}}=\omega_{k} \wedge \tilde{u}_{k}$. The Lamb vector has a large importance in various aspects of fluid dynamics, such as drag and lift reduction in aerodynamics [25, 26], in turbulence [27-29] as well as in aeroacoustics, see [30] for instance. For Beltrami flows where the vorticity vector and the velocity vector are parallel to each other, i.e. when $\mathcal{L}_{B_{k}}=0,\left(\nabla \wedge \tilde{u}_{k} \propto \tilde{u}_{k}\right)$, and for irrotational flows, $\nabla \wedge \tilde{u}_{k}=0$, the first volumetric integral on the right hand side of Equation 22 is reformulated into surface integrals via the extended Green-Gauss theorem. Hence, Equation 22 reduces to:

$$
\begin{aligned}
\int_{\Omega_{g} \cup \Omega_{l}} \tilde{u} \partial_{t} \bar{\rho} d \Omega & =-\sum_{j=i n} \sum_{k=g, l} \int_{\partial \Omega_{j}} \rho_{k} \bar{\alpha}_{k}\left(\tilde{u}_{k} \otimes \tilde{u}_{k}-\frac{\left|\tilde{u}_{k}\right|^{2}}{2} I_{d}\right) d \vec{S}_{j} \\
& -\int_{\sigma}[[\rho \bar{\alpha}]] \tilde{u}_{\sigma} \otimes \tilde{u}_{\sigma} \hat{n}_{\sigma} d \sigma
\end{aligned}
$$


The continuity of the velocities at the interface is assumed in the jump term of Equation 23. For non Beltrami flows, the standard vectorial formulation allows for the Lamb vector $\mathcal{L}_{B_{k}} \neq 0$ to be written as:

$$
\mathcal{L}_{B_{k}}=\omega_{k} \wedge \tilde{u}_{k}=\nabla \cdot\left(\tilde{u}_{k} \otimes \tilde{u}_{k}-\frac{\left|\tilde{u}_{k}\right|^{2}}{2} I_{d}\right)-\tilde{u}_{k}\left(\nabla \cdot \tilde{u}_{k}\right)
$$

For incompressible phases, incorporating the $\mathcal{L}_{B_{k}}$ expression above in the Equation 22, the approximate local momentum reduces to:

$$
\begin{aligned}
\int_{\Omega_{g} \cup \Omega_{l}} \tilde{u} \partial_{t} \bar{\rho} d \Omega & =-\sum_{k=g, l} \int_{\Omega_{k}}\left(\tilde{u}_{k} \otimes \tilde{u}_{k}-\frac{\left|\tilde{u}_{k}\right|^{2}}{2} I_{d}\right) \nabla \rho_{k} \bar{\alpha}_{k} d \Omega_{k} \\
& -\int_{\sigma}[[\rho \bar{\alpha}]] \frac{\left|\tilde{u}_{\sigma}\right|^{2}}{2} I_{d} \hat{n}_{\sigma} d \sigma
\end{aligned}
$$

The volumetric terms appearing in the Equation 25 is a dyadic force resulting from density and volume fraction gradients while the second force is an internal force on the interface, it results from the change in kinetic energy due to the jump in density and volume fraction across the interface.

\subsection{Convective momentum on bend}

The evaluation of convective forces requires a reformulation of the balance laws in the framework of averaging procedures that distinguish large scales from small ones, see equation C.3 in Appendix C. The fluctuating components of FavreReynolds stress $\mathcal{R}_{\mathcal{F}}$ were cast into the momentum balance. The first component $\mathcal{R}_{s}=\overline{u^{\prime} \otimes u^{\prime}}$, is the standard Reynolds stress, and the second component that is labelled $\mathcal{R}_{c}$ (see Appendix C), is an immediate consequence of compressibility; it is turbulent mass flow dependent and relates to variable density effects.

Regrouping averaged and fluctuating momentum terms from inlet and outlet sides, 
with vanishing convective fluxes on the lateral surface $\partial \Omega_{L}$, leads to:

$$
\begin{aligned}
\int_{\Omega_{l} \cup \Omega_{g}} \nabla \cdot\left(\bar{\rho} \tilde{u} \otimes \tilde{u}+\bar{\rho} \mathcal{R}_{\mathcal{F}}\right) d \Omega & =\sum_{j=\text { in }} \sum_{k=g, l} \int_{\partial \Omega_{j}} \rho_{k} \bar{\alpha}_{k}\left(\tilde{u}_{k} \otimes \tilde{u}_{k}+\mathcal{R}_{\mathcal{F}}^{k}\right) d \vec{S}_{j} \\
& +\int_{\sigma}\left([[\rho \bar{\alpha}]] \cdot \tilde{u}_{\sigma} \otimes \tilde{u}_{\sigma}+\left[\left[\rho \bar{\alpha} \mathcal{R}_{\mathcal{F}}^{\sigma}\right]\right]\right) \hat{n}_{\sigma} d \sigma
\end{aligned}
$$

where $k=g, l$ and $\sigma$. The continuity of averaged tangential and transverse velocities across the interface is assumed, i.e., $\tilde{u}_{g}=\tilde{u}_{l}=\tilde{u}_{\sigma}$. The force includes the jump in the Favre-Reynolds stress $\mathcal{R}_{\mathcal{F}}^{k}$ at the interface:

$$
\mathcal{R}_{\mathcal{F}}^{k}=\overline{u_{k}^{\prime} \otimes u_{k}^{\prime}}+\frac{\overline{\alpha_{k}^{\prime} u_{k}^{\prime} \otimes u_{k}^{\prime}}}{\bar{\alpha}_{k}}-\frac{\overline{\alpha_{k}^{\prime} u_{k}^{\prime}} \otimes \overline{\alpha_{k}^{\prime} u_{k}^{\prime}}}{\bar{\alpha}_{k}^{2}}
$$

This expression in its actual form obscures its analysis due to the presence of high order statistics and mixed moments. To shun this hurdle, a bounded approximation is derived in Appendix C through Grüss-type-inequality.

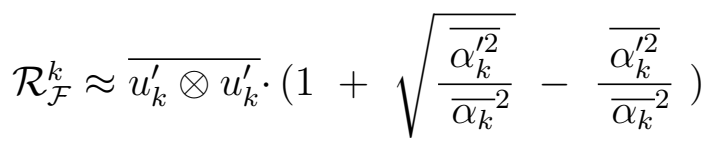

The equality holds for flows with measurable fluctuating moments obeying to non-skewed distributions, i.e statistics of the variables are such that $\psi_{\min }^{\prime} \approx$ $-\psi_{\text {max }}^{\prime}$, which presumably corresponds to compressible turbulent flows at moderate Reynolds number. Researchers [31] confirmed that the data from DNS showed a transition in skewness with an increase in Mach number, while in general the effect of Reynolds number is weak.

Departures from (incompressible) standard Reynolds stress $\mathcal{R}_{s}$ result purely from void fraction (or density) fluctuations. The scales of these departures depend on the conditions and on the side of the interface. This is of importance in the vicinity of the interface where substantial fluctuations inceptions imply redistributions in turbulent fluxes and kinetic energy. The expression of $\mathcal{R}_{\mathcal{F}}^{k}$ shows that void fraction fluctuations effects materialize with conflicting contributions of first and second orders. 


\subsection{Elbow forces description}

The mechanical force exerted on the elbow can be evaluated through Computational Fluid Dynamics (CFD). The mathematical structure of the total surface forces $\vec{F}_{S}$ per unit surface is required in order to understand how these forces should be represented. An important statement related to the stress tensor is that the force per unit area exerted by the fluid on a surface, with unit normal $\hat{n}_{S}$ pointing into the fluid, can be expressed in the present framework by:

$$
\begin{aligned}
\vec{F}_{S} & =\mathcal{T} \cdot \hat{n}_{S} \\
& =\left(-p \cdot I_{d}+\mu \cdot\left(\nabla \vec{u}+\nabla \vec{u}^{T}\right)+\lambda(\nabla \cdot \vec{u}) \cdot I_{d}\right) \cdot \hat{n}_{S}
\end{aligned}
$$

where, $\hat{n}_{S}$ is the outward unit normal vector to the elementary surface $d S$. The mechanical pressure $p_{m}$ exerted on the unit surface is defined as the averaged normal stress, also known as the first invariant of the stress tensor. It is related to the pressure $p$ as follows, with $t r$ being the trace of the matrix $\mathcal{T}$ :

$$
\begin{aligned}
p_{m} & =-\frac{1}{3} \operatorname{tr}(\mathcal{T}) \\
& =p-\eta \nabla \cdot \vec{u}
\end{aligned}
$$

The two pressures are equivalent for incompressible fluids: $\nabla \cdot \vec{u}=0$, or under Stokes' hypothesis: $\eta=0$. Thermodynamically, this is equivalent to the reversibility of the processes (thermodynamic paths). This requirement implies that the fluid does not dissipate energy under deformations. The expressions of the projected components of a force $\vec{F}_{S}$ exerted on a unit surface $d S$ with outward unit normal $\hat{n}_{S}$ that is defined by its local orthonormal components $\left(n_{S x}, n_{S y}, n_{S z}\right)$ are given by:

$$
\left\{\begin{array}{l}
F_{S x}=\left.\mathcal{T} \cdot \hat{n}_{S}\right|_{x}=\left(-p+\lambda \nabla \cdot \vec{u}+\tau_{x x}\right) \cdot n_{S x}+\tau_{x y} \cdot n_{S y}+\tau_{x z} \cdot n_{S z} \\
F_{S y}=\left.\mathcal{T} \cdot \hat{n}_{S}\right|_{y}=\tau_{y x} \cdot n_{S x}+\left(-p+\lambda \nabla \cdot \vec{u}+\tau_{y y}\right) \cdot n_{S y}+\tau_{y z} \cdot n_{S z} \\
F_{S z}=\left.\mathcal{T} \cdot \hat{n}_{S}\right|_{z}=\tau_{z x} \cdot n_{S x}+\tau_{z y} \cdot n_{S y}+\left(-p+\lambda \nabla \cdot \vec{u}+\tau_{z z}\right) \cdot n_{S z}
\end{array}\right.
$$


The resultant force $F_{S}$ is simply the Euclidean norm formed by the above components.

\section{Simulation results}

In order to generate slug flows, three simulations were performed separately in a horizontal pipe of length $L=30 \mathrm{~m}$ and diameter $D=0.078 \mathrm{~m}$ at atmospheric pressure, with fluids properties shown in Table 1 and initial conditions of mixture flow rates specified in Table 2. According to the flow map in [32], the initial flow conditions for Cases 1-3 in Table 2 correspond to a slug flow regime. A mesh sensitivity analysis was performed on this horizontal section to check that numerical slug frequencies were in agreement with experimental-based ones [33].

\begin{tabular}{|l|l|l|}
\hline \hline Fluids & Density $\left[\mathrm{kg} / \mathrm{m}^{3}\right]$ & Viscosity $[\mathrm{Pa} \mathrm{s}]$ \\
\hline Air & 1.225 & $1.78910^{-5}$ \\
\hline Water & 998.2 & $1.00410^{-3}$ \\
\hline \hline
\end{tabular}

Table 1: Fluids Physical properties

\begin{tabular}{|l|l|l|}
\hline \hline Case reference & $\begin{array}{l}\text { Gas mass flow } Q_{g} \\
{[\mathrm{~kg} / \mathrm{s}]}\end{array}$ & $\begin{array}{l}\text { Water mass flow } \\
Q_{l}[\mathrm{~kg} / \mathrm{s}]\end{array}$ \\
\hline Case-1 & 0.025 & 2.48 \\
\hline Case-2 & 0.0493 & 2.87 \\
\hline Case-3 & 0.0495 & 4.97 \\
\hline \hline
\end{tabular}

Table 2: Initial flow conditions in a linear $30 \mathrm{~m}$ long pipe

Flow data extracted $27 \mathrm{~m}$ far from the inlet, were recorded at each time step of the flow solver $\left(10^{-3} \mathrm{~s}\right)$ during the simulation. These data, including velocity, volume fraction and turbulence properties obtained for fully established slug flows have been used as inlet conditions of the elbow system considered in this work. 
A similar approach was described by the authors in [17] for the analysis of slugs into helical pipes.

The elbow system considered here has the same diameter as for the horizontal section, i.e. $D=0.078 \mathrm{~m}$. Upstream and downstream horizontal sections from the elbow have lengths of $l_{u}=2 \mathrm{~m}$ and $l_{d}=1.15 \mathrm{~m}$ respectively, and the elbow curvature presents a mid-radius of $R=0.0126 \mathrm{~m}$. A structured mesh of $\approx 1.23 \mathrm{M}$ cells comprising $\approx 900$ cross-sectional and 64 circumferential cells was built as shown in figure 1.

Similarly to the horizontal section, velocity-inlet and Pressure outlet boundary conditions were considered at the inlet and outlet sections of the elbow system. The pressure-based solver (segregated solver) was selected to run the transient slug flow simulations. All numerical schemes applied for the VOF-based simulations are listed in Table 3.

\begin{tabular}{|c|c|c|c|}
\hline \multicolumn{4}{|c|}{ Transient VOF Solver } \\
\hline Pressure-Velocity & Spatial Discret. & Pressure & Momentum \\
\hline Simple & $\begin{array}{l}\text { Least Squares } \\
\text { Cell Based }\end{array}$ & Presto & $2^{\text {nd }}$ Order Upwind \\
\hline Volume Fraction & $\begin{array}{l}\text { Turbulence } \\
\text { Kinetic rate }\end{array}$ & $\begin{array}{l}\text { Turbulence Dis- } \\
\text { sipation rate }\end{array}$ & $\begin{array}{l}\text { Transient } \\
\text { Formulation }\end{array}$ \\
\hline Modified HRIC & $\begin{array}{l}2^{\text {nd }} \text { Order Up- } \\
\text { wind }\end{array}$ & $\begin{array}{l}2^{\text {nd }} \text { Order Up- } \\
\text { wind }\end{array}$ & $1^{\text {st }}$ Order Implicit \\
\hline
\end{tabular}

Table 3: Volume of fluid solver

The $R N G k-\epsilon$ model, described previously in Section 2.2 was selected along with non-equilibrium wall functions to capture turbulence effects with a bounded value of $50<y^{+}<130$ obeying the ANSYS Fluent user's guide [21] recommendation. 


\subsubsection{Case 1}

Case 1 corresponds to the lowest mixture flow rates; results for this case are shown in Figure 3. The instantaneous profiles of liquid hold up and flow rate displayed in this picture indicate a slugging regime at the inlet of the elbow, with maximum peaks around $26 \mathrm{~kg} / \mathrm{s}$. The regime is inherently unstable and it is not straightforward to distinguish between forces due to slugs, to splashes or resulting from waves. Subsequently, the analysis of the fluctuating pressures and stresses based on a visual analysis is incredibly complicated.

Looking at the peak forces signals in an attempt to attribute these to slug bodies impacting the outer wall of the elbow or to identify those due to waves from those due to sliding liquid films on the outer bend wall during the inception of "film inversion" remains vain. 


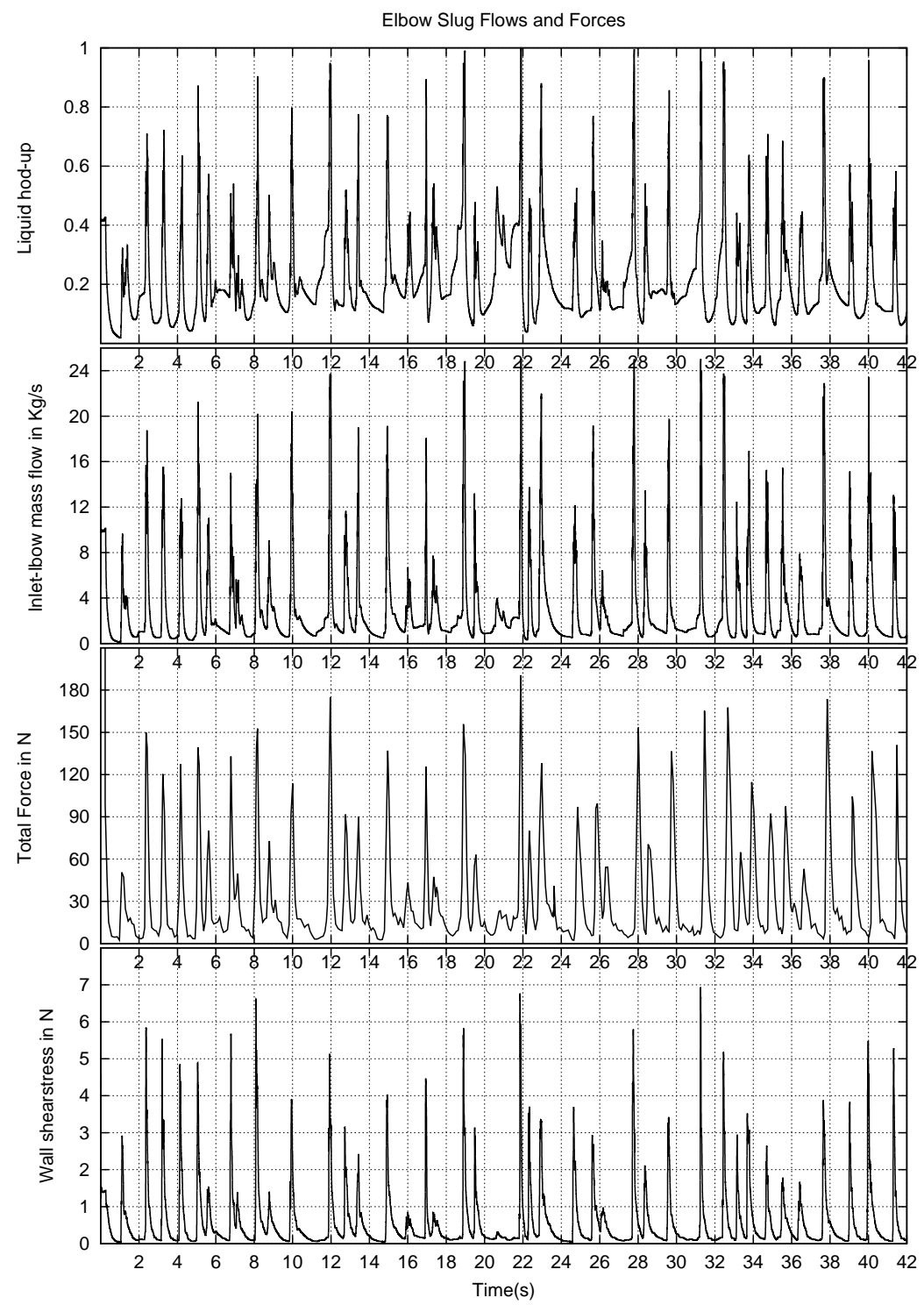

Figure 3: Inlet mass flow rates and forces in the bend - Case 1

To be able to interpret the results shown in Figure 3, it is crucial to identify slug entities during the flow. To estimate slug frequencies, it is a common practice to define a criterion based on the liquid hold-up. This approach has also been applied here and each peak of liquid hold-up $\alpha_{l} \geq 0.7$ has been accounted for as a slug. In the present case, this corresponds roughly to peaks of mixture flow 
rates $Q_{m i x} \geq 18 \mathrm{~kg} / \mathrm{s}$. Based on the above criterion, the magnitude of the forces due to slug body impacts is usually higher than $120 \mathrm{~N}$. There are however slugs exerting lower impacts, around $90 \mathrm{~N}$, for example peaks at times $t=13.5 \mathrm{~s}$ and $t=26 \mathrm{~s}$. These lower magnitudes can be attributed to unstable slugs which have a propensity to collapse or to the limitation of the liquid hold-up criterion established to identify slug impacts.

\subsubsection{Case 2}

Simulation results for Case 2 are shown in Figure 4. These plots depict forces with magnitudes and a base line of liquid hold up larger than those exhibited in Case 1. Based on the liquid volume fraction threshold criterion used previously, slugs are accounted for when peaks of mixture mass flow rates are such that $Q_{m i x} \geq 22 \mathrm{~kg} / \mathrm{s}$. In the present case, the maximum operating loads are higher than $280 \mathrm{~N}$ and slug impacts show forces with magnitudes in the vicinity of $200 \mathrm{~N}$. As for the previous case, several slug impacts display magnitude forces lower than $160 \mathrm{~N}$, although they are accounted for as slugs, see peaks at $t=14.25 \mathrm{~s}, t=15 \mathrm{~s}$ and $t=22 \mathrm{~s}$ for instance.

It appears clearly that the above paradox results from the deficiency of the criterion based on the liquid hold-up. Using solely this criterion seems not to be sufficient to distinguish big waves from slugs. In order to narrow the identification of forces due to slugs, the criterion has to be supplied with a condition on slug translational velocities. In an Eulerian formalism, mixture velocities are accessible while slug translational velocities $u_{T S}$ (which are Lagrangian) are difficult to evaluate, especially for 3D transient models. 


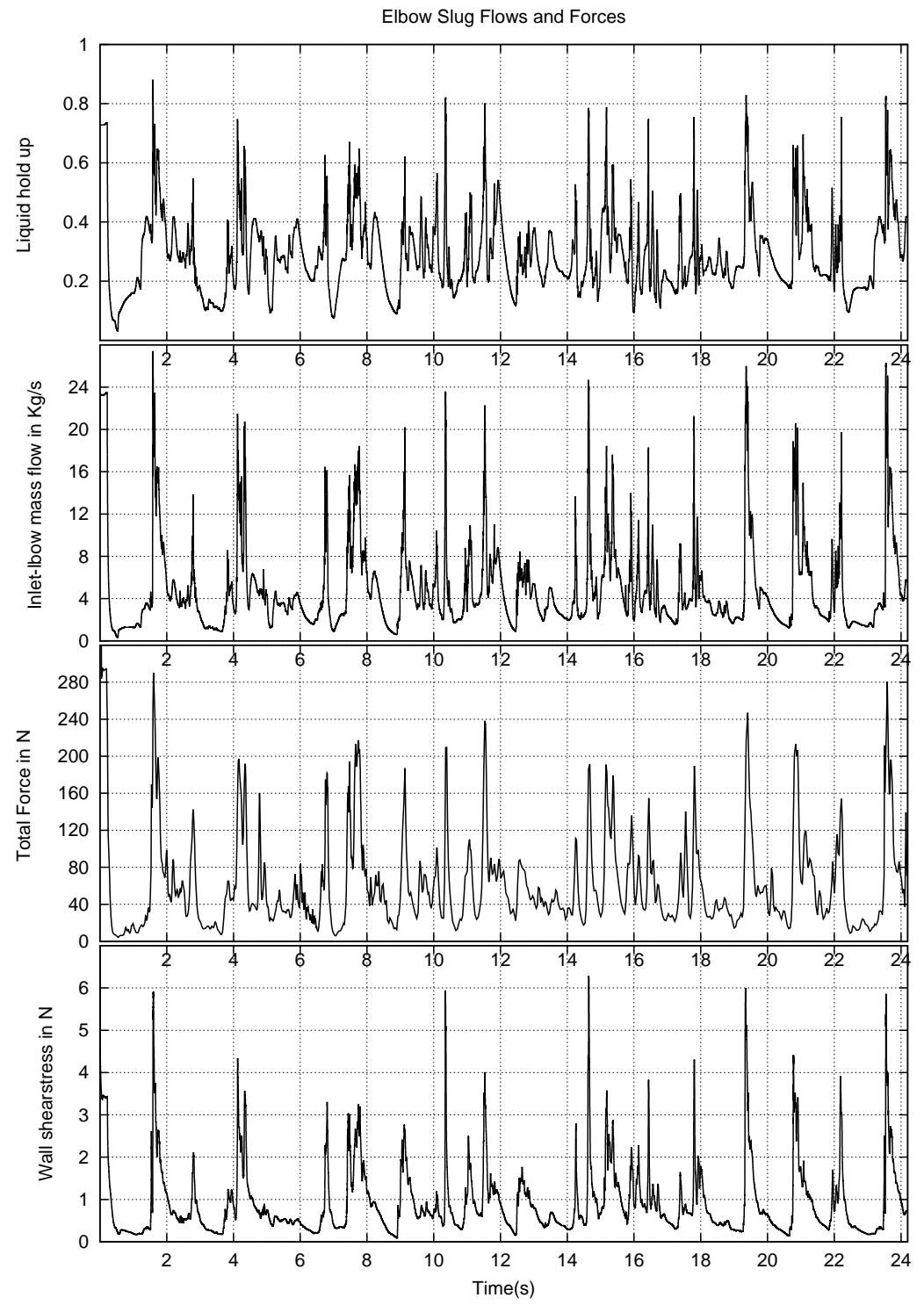

Figure 4: Inlet mass flow rates and forces in the bend - Case 2

The correlation from [34] could potentially be used as a supplementary criterion:

$$
\begin{aligned}
u_{T S} & =1.2 \cdot u+0.54 \cdot \sqrt{g D} \\
\text { with } \quad u & =\alpha_{l} \cdot u_{l}+\alpha_{g} \cdot u_{g}
\end{aligned}
$$

However, the use of such criterion is possible when the mixture velocity $u$ is 
known, i.e. in the case of a slug flow regime, when all slugs are identical; this is rarely the case in industrial applications. Such remark is in agreement with the liquid hold-up trace shown here where it appears clearly that all slugs are far from being regular or identical.

It can also be noticed that the time trace exhibits peaks of resultant force larger than $200 \mathrm{~N}$, see peaks at times $t=7.9 \mathrm{~s}$ and $t=21 \mathrm{~s}$. Although their corresponding liquid hold-up extrema are not recognized as slugs, these resultant forces seem to result from the merging of a number of waves.

\subsubsection{Case 3}

Results for Case 3 are shown in Figure 5. The liquid flow rate is much higher than in the two previous cases. The traces of liquid hold-up indicate that most of the slugs traveling into the elbow are preceded by large liquid pools $\alpha_{l} \geq 0.35$. This implies that the flow line is continuously pre-loaded. Based on the hold-up threshold criterion $\alpha_{l} \geq 0.7$, peaks of mixture mass flow rates such that $Q_{\text {mix }} \geq$ $24 \mathrm{~kg} / \mathrm{s}$ are identified as being slugs. The maximum loading in this case reaches magnitudes higher than $300 N$ while slug bodies depict impacts of magnitude higher than $250 \mathrm{~N}$.

In all three test cases investigated, the time traces of shear-stress exhibit resultant loads with magnitudes less than $5.5 \%$ of the total loads due to isotropic stresses. The time traces of resultant loads contain both compressive and tensional components. As expected, the magnitude of resultant forces increases with the increase of mixture flow rates.

Slug flows investigated here exhibit slug frequencies of $\omega_{1}=0.40 \mathrm{~s}^{-1}, \omega_{2}=$ $0.45 s^{-1}$ and $\omega_{3}=0.54 s^{-1}$ for Cases 1, 2 and 3, respectively. These frequencies were established through counting the number of slugs present during a period of time, here, $30 \mathrm{~s}$. As can be observed from these cases, the resultant loading cycle is much higher than the slugging cycle, while the severity (maxima) of the load is 
mainly due to slug bodies.

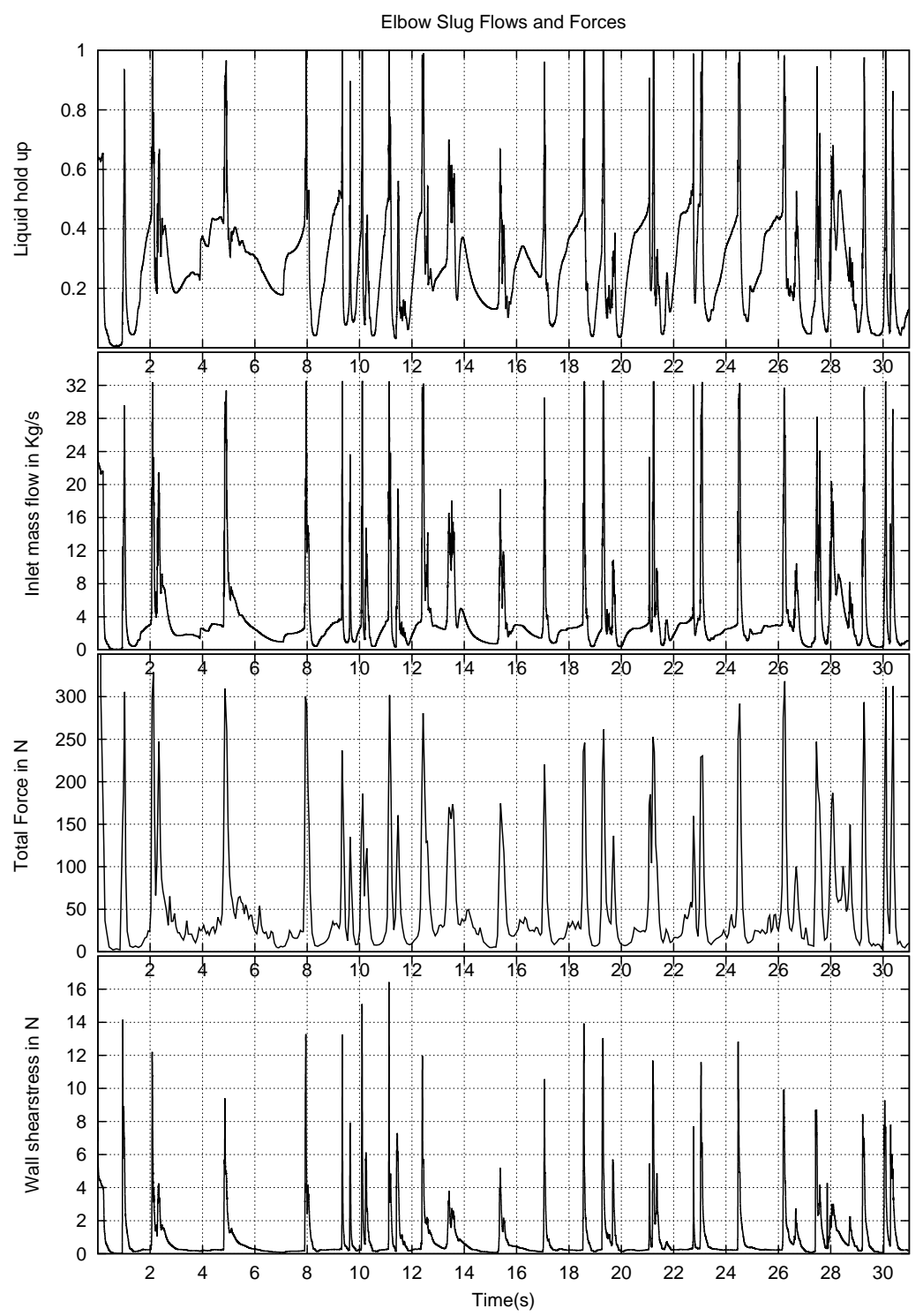

Figure 5: Inlet mass flow rates and forces histograms in the bend - Case 3

A liquid film inversion in elbows or in coiled pipes is often observed at low liquid flow rates. During these occurrences, centrifugal forces prevail to control the redistribution of the phases. Such liquid film inversion and resulting distribution 
of volume fraction for both phases are shown in Figure 6 for Case 3 at $t=13 \mathrm{~s}$. It can be seen that a transition from a stratified to a core annular flow regime took place in the elbow.
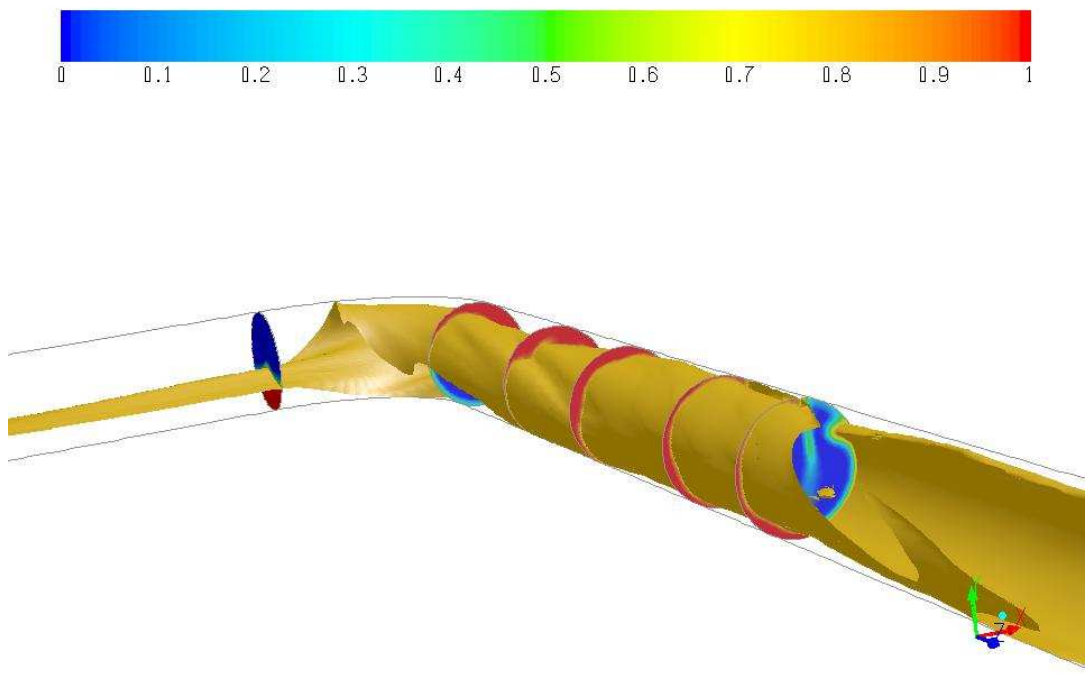

Figure 6: Film inversion at time $\mathrm{t}=13 \mathrm{~s}$ : transition from stratified to annular flow in the bend; pure liquid (red colour), pure gas (blue colour), liquid-gas interface (gold colour)

\section{Conclusion}

This study considered established two-phase gas/liquid slug flows in a horizontal elbow displaying a continuous singular interface. The volumetric forces budget due to the mixture were obtained through the use of the VOF model. The distributed Newtonian and Configurational forces expressions were derived. Contact line force due to surface tension and forces due to jump conditions across the interface were provided.

The proposed approach uses time scales separation to evaluate the time rate of local momentum. Neglecting the contribution of the time rate of mean mixture velocity and keeping only the contribution due to the time rate of the volume 
fraction allows for a derivation showing a close connection between the Lamb vector (vortex-force) and the time rate acceleration. To the authors' best knowledge, such novel procedure has never been reported previously.

Through the use of Grüss-inequalities, it was possible to provide a useful expression that formulate the deviation of Favre-Reynolds stress from the standard Reynolds stress. The expression materializes the involvement of fluctuating void fraction (compressibility) in the mentioned deviation with conflicting contributions of first and second order (in void fraction fluctuation) and in addition, this emphasizes the changes in turbulence across the interface.

Exerted Newtonian forces of a slug flow on a bend were estimated through CFD. The study showed the increase of the magnitude of the force maxima with the increase of the mixture flow rates, as expected. The resultantes forces obtained here are one to two orders higher in magnitude than the maximum forces obtained with PFM model presented in [5]. The actual resultants are however well in the same range as the forces measured and predicted in [7]. Nevertheless, a criterion based solely on the liquid hold-up threshold has proved to be insufficient to distinguish forces due to slug impacts from those due to liquid films and waves. Identifying individual slugs when travelling through a bend is therefore essential. The use of a Lagrangian criterion, equivalent to that of [34], which is based on slug translational velocity, would be useful to correlate slugs and impacts. Such procedure was successfully applied in [35], where the authors have used two probes located at close proximity, hence they were able to measure slug translational velocities, which in turn were correlated to CFD with high accuracy.

The numerical results also showed loading cycles much larger than slugging cycles, which is important from a dynamical and a structural integrity point of view.

Finally, the time traces have highlighted the importance of the static pressure 
over the shear stress. The marginal contributions of viscous shear-stresses on the resultant force was clearly highlighted in all tests presented in this study.

\section{A. Appendix.}

For the derivation of the jump law in the following, the field $\psi$ is considered in term of distributions. $\psi$ is considered as a smooth continuous quantity except in the vicinity of the singular interface $\sigma$ where it possesses a finite discontinuity, see Figure 7, $\psi_{k}(s)=\lim _{s \rightarrow \sigma_{k}} \psi(s)$ with $k=g, l$.

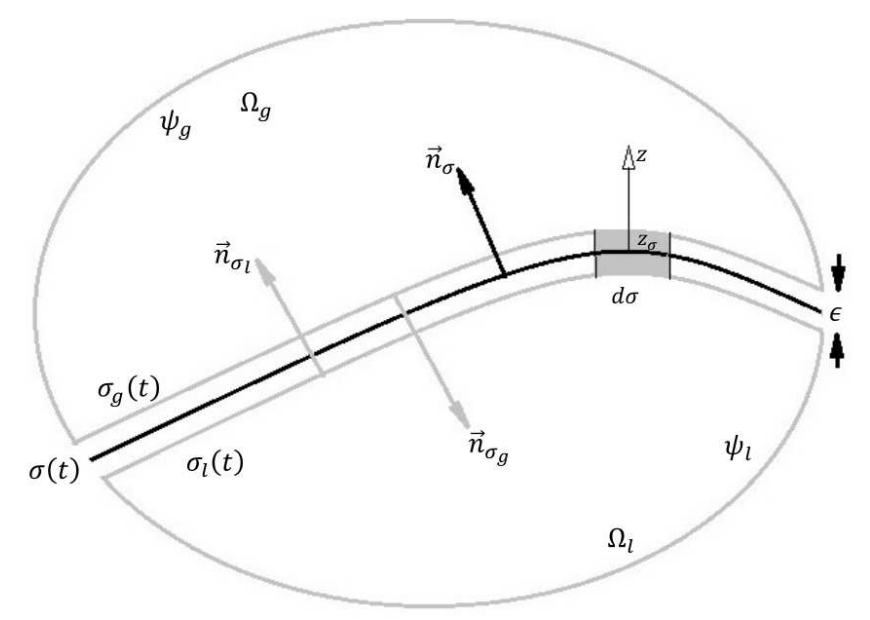

Figure 7: Schematic representation of the moving singular interface $\sigma(t)$

To regularize the variable $\psi(s)$, two transition sub-layers $\gamma_{k}$, with $k=g, l$ are formed by sweeping the elements of $\sigma$ in the directions $\vec{n}_{\sigma}$ and $-\vec{n}_{\sigma}$. Based on the convention used in Figure $7, \vec{n}_{\sigma_{l}}=-\vec{n}_{\sigma_{g}}=\vec{n}_{\sigma}$. The obtained sub-layers $\gamma_{k}$ are enclosed by $\sigma$ and $\sigma_{k} \equiv \sigma \pm \epsilon / 2$. The Green-Gauss theorem applied to $\psi$ in the sub-region $\gamma_{g} \cup \gamma_{l}$ becomes:

$$
\int_{\gamma_{g} \cup \gamma_{l}} \partial_{z} \psi d\left(\gamma_{l} \cup \gamma_{g}\right)=\int_{\sigma} \int_{-\epsilon / 2}^{+\epsilon / 2} \partial_{z} \psi d \eta d \sigma
$$

The volume element is $d \eta d \sigma=d \eta d \sigma_{k}$ with $\eta=z-z_{\sigma}, z_{\sigma}$ located at the surface element $d \sigma$ and $z \in[-\epsilon / 2,+\epsilon / 2]$. 
The extension of the field $\psi_{g}$ to $\gamma_{l}$ and $\psi_{l}$ to $\gamma_{g}$ can be achieved via the smoothed Heaviside step function $\hat{h}(z)$ :

$$
\hat{h}(z)= \begin{cases}1 & \text { if } z \geq z_{\sigma}+\epsilon / 2 \\ 1 / 2 & \text { if } z=z_{\sigma} \\ 0 & \text { if } z \leq z_{\sigma}-\epsilon / 2\end{cases}
$$

The Green-Gauss theorem applied to regularised variable becomes:

$$
\begin{aligned}
\int_{\sigma} \int_{-\epsilon / 2}^{+\epsilon / 2} \partial_{z} \psi d \eta d \sigma & =\int_{\sigma} \int_{-\epsilon / 2}^{+\epsilon / 2} \partial_{z}\left[\psi_{l} \hat{h}(z)+\psi_{g} \hat{h}(-z)\right] d \eta d \sigma \\
& =\int_{\sigma} \int_{-\epsilon / 2}^{+\epsilon / 2}\left[\hat{h}(z) \partial_{z} \psi_{l}+\hat{h}(-z) \partial_{z} \psi_{g}\right] d \eta d \sigma \\
& +\int_{\sigma} \int_{-\epsilon / 2}^{+\epsilon / 2}\left(\psi_{l}-\psi_{g}\right) \partial_{z} \hat{h}(z) d \eta d \sigma \\
& =\int_{\gamma_{l}} \partial_{z} \psi_{l} d \gamma_{l}+\int_{\gamma_{g}} \partial_{z} \psi_{g} d \gamma_{g} \\
& +\int_{\sigma} \int_{-\epsilon / 2}^{+\epsilon / 2}\left(\psi_{l}-\psi_{g}\right) \partial_{z} \hat{h}(z) d \eta d \sigma
\end{aligned}
$$

The Heaviside function derivative $\hat{h}(z)$ relates to the Dirac $\delta$ distribution by $\partial_{z} \hat{h}(z)=\delta_{\epsilon}(\sigma) \cdot \hat{n}_{\sigma}$ and $\lim _{\epsilon \rightarrow 0} \delta_{\epsilon}(\sigma)=\delta(\sigma)$. This complete the jump law, thus:

$$
\begin{aligned}
\lim _{\epsilon \rightarrow 0} \int_{\gamma_{l} \cup \gamma_{l}} \partial_{z} \psi d\left(\gamma_{l} \cup \gamma_{l}\right) & =\lim _{\epsilon \rightarrow 0} \int_{\sigma} \int_{-\epsilon / 2}^{+\epsilon / 2}\left(\psi_{l}-\psi_{g}\right) \delta_{\epsilon}(\sigma) \cdot \hat{n}_{\sigma} d \eta d \sigma \\
& =\int_{\sigma}[[\psi]] \cdot \hat{n}_{\sigma} d \sigma
\end{aligned}
$$

where the generic jump across $\sigma$ is $[[\psi]]=\psi_{l}-\psi_{g}$.

\section{B. Appendix}

The mixture velocity $u$ is considered under the hypothesis of separated fluids, hence $\lim _{\alpha_{k} \rightarrow 1} u=u_{k}$, and for the mixture density $\rho, \lim _{\alpha_{k} \rightarrow 1} \rho=\rho_{k}$. In the following, 
$\bar{\rho}_{k}=\rho_{k} \bar{\alpha}_{k}$ stands for the effective density of the phase $k$.

$$
\begin{aligned}
\int_{\Omega_{g} \cup \Omega_{l}} \tilde{u} \partial_{t} \bar{\rho} d \Omega & =-\int_{\Omega_{g} \cup \Omega_{l}} \tilde{u} \nabla \cdot\left(\rho_{g} \overline{\alpha_{g}}+\rho_{l} \overline{\alpha_{l}}\right) \tilde{u} d \Omega \\
& =-\sum_{k=g, l} \int_{\Omega_{k}} \tilde{u_{k}} \nabla \cdot \bar{\rho}_{k} \tilde{u}_{k} d \Omega_{k} \\
& =-\sum_{k=g, l} \int_{\Omega_{k}}\left(\nabla \cdot \tilde{u}_{k} \otimes \bar{\rho}_{k} \tilde{u}_{k}-\left(\bar{\rho}_{k} \tilde{u}_{k} \cdot \nabla\right) \tilde{u}_{k}\right) d \Omega_{k} \\
& =-\sum_{k=g, l} \int_{\Omega_{k}}\left(\nabla \cdot \tilde{u}_{k} \otimes \bar{\rho}_{k} \tilde{u}_{k}-\bar{\rho}_{k}\left(\nabla \frac{\left|\tilde{u}_{k}\right|^{2}}{2}-\tilde{u}_{k} \wedge \omega_{k}\right)\right) d \Omega_{k}
\end{aligned}
$$

Through the above derivation, vectorial identities $\psi \nabla \cdot \psi=\nabla \cdot \psi \otimes \psi-(\psi \cdot \nabla) \psi$ and $(2 \psi \cdot \nabla) \psi=\nabla|\psi|^{2}-2 \psi \wedge(\nabla \wedge \psi)$ are used. The symbol $\otimes$ represents the dyadic product. The last term contains the vorticity $\omega_{k}=\nabla \wedge \tilde{u}_{k}$.

Expanding the vorticity term and using the relation $\nabla\left|\psi_{k}\right|^{2}=\nabla \cdot\left(\left|\psi_{k}\right|^{2} I_{d}\right)$ yields.

$$
\begin{aligned}
\int_{\Omega_{g} \cup \Omega_{l}} \tilde{u} \partial_{t} \bar{\rho} d \Omega & =-\sum_{k=g, l} \int_{\Omega_{k}} \nabla \cdot \rho_{k} \bar{\alpha}_{k}\left(\tilde{u}_{k} \otimes \tilde{u}_{k}-\frac{\left|\tilde{u}_{k}\right|^{2}}{2} I_{d}\right) d \Omega_{k} \\
& -\sum_{k=g, l} \int_{\Omega_{k}} \frac{\left|\tilde{u}_{k}\right|^{2}}{2} I_{d} \nabla\left(\rho_{k} \bar{\alpha}_{k}\right) d \Omega_{k}+\sum_{k=g, l} \int_{\Omega_{k}} \rho_{k} \bar{\alpha}_{k} \omega_{k} \wedge \tilde{u}_{k} d \Omega_{k}
\end{aligned}
$$

The second integral on the right hand side of Equation B.2 simplifies to the surface integral over the singular interface, where the density and volume fraction gradients are significant, hence:

$$
\begin{aligned}
\int_{\Omega_{g} \cup \Omega_{l}} \tilde{u} \partial_{t} \bar{\rho} d \Omega & =-\sum_{k=g, l} \int_{\Omega_{k}} \nabla \cdot \rho_{k} \bar{\alpha}_{k}\left(\tilde{u}_{k} \otimes \tilde{u}_{k}-\frac{\left|\tilde{u}_{k}\right|^{2}}{2} I_{d}\right) d \Omega_{k} \\
& -\int_{\sigma} \frac{\left|\tilde{u}_{\sigma}\right|^{2}}{2} I_{d}[[\rho \bar{\alpha}]] \hat{n}_{\sigma} d \sigma_{k}+\sum_{k=g, l} \int_{\Omega_{k}} \rho_{k} \bar{\alpha}_{k} \omega_{k} \wedge \tilde{u}_{k} d \Omega_{k}
\end{aligned}
$$




\section{Appendix}

Continuity and momentum balance laws for compressible turbulent flows are briefly provided here. For detailed derivations, see [36]. Reynolds and Favre averaging methods are usual starting points for turbulence evaluations. Instantaneous flow variables $\psi$ are split into $\bar{\psi}$, averaged and fluctuating Reynolds parts $\psi^{\prime}$. Also, let $\widehat{\psi}$ be a normalized turbulent quantity, hence:

$$
\begin{aligned}
& \psi=\bar{\psi}+\psi^{\prime} \\
& \widehat{\psi}=\overline{\rho^{\prime} \psi^{\prime}} / \bar{\rho}
\end{aligned}
$$

Favre averaged and fluctuating components counter parts of $\psi$ are:

$$
\psi=\tilde{\psi}+\psi " \text { with } \quad \tilde{\psi}=\overline{\rho \psi} / \bar{\rho}
$$

Equally, dynamic and bulk viscosities of fluids expand to $\mu=\bar{\mu}+\mu^{\prime}$ and $\eta=\bar{\eta}+\eta^{\prime}$. Under the above procedures, the conservation laws 1 and 2 may now be recast.

$$
\begin{aligned}
& \partial_{t} \bar{\rho}+\nabla \cdot(\bar{\rho} \bar{u}+\bar{\rho} \widehat{u})=0 \\
& \partial_{t} \bar{\rho} \tilde{u}+\nabla \cdot(\bar{\rho} \tilde{u} \otimes \tilde{u}+\overline{\rho u " \otimes u "})=\nabla \cdot \overline{\mathcal{T}}+\bar{\rho} \cdot \vec{g}+\bar{F}
\end{aligned}
$$

The mean stress $\overline{\mathcal{T}}=\mathcal{T}\left(\bar{p}, \bar{\tau}+\overline{\tau^{\prime}}\right)$ is linear with respect to averaging procedure. Ignoring fluctuations in viscosities leads to further simplification, i.e $\overline{\tau^{\prime}}=0$. With

the use of $\widetilde{\psi}-\bar{\psi}=\psi^{\prime}-\psi "=\widehat{\psi}$, the reformulation of the fluctuating convective term, the Favre-Reynolds stress $\mathcal{R}_{\mathcal{F}}$ yields:

$$
\begin{aligned}
\bar{\rho} \mathcal{R}_{\mathcal{F}} & =\overline{\rho u^{\prime \prime} \otimes u^{\prime \prime}} \\
& =\underbrace{\bar{\rho} \overline{u^{\prime} \otimes u^{\prime}}}_{\bar{\rho} \mathcal{R}_{s}}+\underbrace{\overline{\rho^{\prime} u^{\prime} \otimes u^{\prime}}-\bar{\rho} \otimes \widehat{u}}_{\bar{\rho} \mathcal{R}_{c}} \\
& =\underbrace{\bar{u}}
\end{aligned}
$$

with $\mathcal{R}_{s}$ being the standard Reynolds stress and $\mathcal{R}_{c}$, termed here for a compressible Reynolds stress, relates to compressibility and variable density of the flow. 
Incompressible fluids were considered here, thus the compressibility is shifted to the volume fractions, $\alpha_{k}=\bar{\alpha}_{k}+\alpha_{k}^{\prime}$; with $k=g, l$, hence:

$$
\bar{\rho}_{k}=\rho_{k} \bar{\alpha}_{k} \quad \text { and } \quad \rho_{k}^{\prime}=\rho_{k} \alpha_{k}^{\prime}
$$

Consequently, the phasic expression of Favre-Reynolds stress $\mathcal{R}_{\mathcal{F}}^{k}$ states:

$$
\mathcal{R}_{\mathcal{F}}^{k}=\overline{u_{k}^{\prime} \otimes u_{k}^{\prime}}+\overline{\overline{\alpha_{k}^{\prime} u_{k}^{\prime} \otimes u_{k}^{\prime}}} \frac{\overline{\alpha_{k}^{\prime} u_{k}^{\prime}} \otimes \overline{\alpha_{k}^{\prime} u_{k}^{\prime}}}{{\overline{\alpha_{k}}}^{2}}
$$

To derive a clearer expression, firstly, the third order statistics term (phasic normalised Reynolds stress $\widehat{\mathcal{R}_{s}^{k}}$ ) is approached by using pre-Grüss-inequality, see [37]. From this point in this appendix, $\Delta_{\psi^{\prime}}=\left(\psi_{\max }^{\prime}-\psi_{\min }^{\prime}\right)$, with $\psi^{\prime}$ denoting a fluctuating quantity.

$$
\left|\overline{\alpha_{k}^{\prime} u_{k}^{\prime} \otimes u_{k}^{\prime}}-\sqrt{\overline{\alpha_{k}^{\prime 2}}} \cdot \overline{u_{k}^{\prime} \otimes u_{k}^{\prime}}\right| \leq \frac{\sqrt{\overline{\alpha_{k}^{\prime 2}}} \cdot \Delta_{u_{k}^{\prime} \otimes u_{k}^{\prime}}}{2}
$$

Secondly, for the fourth order statistics term, the known statistical version of Grüss-inequality [38], is used, hence:

$$
\left|\overline{\alpha_{k}^{\prime} u_{k}^{\prime} \otimes \alpha_{k}^{\prime} u_{k}^{\prime}}-\overline{\alpha_{k}^{\prime} u_{k}^{\prime}} \otimes \overline{\alpha_{k}^{\prime} u_{k}^{\prime}}\right| \leq \frac{\Delta_{\alpha_{k}^{\prime} u_{k}^{\prime}} \otimes \Delta_{\alpha_{k}^{\prime} u_{k}^{\prime}}}{4}
$$

Also one has $\overline{\alpha_{k}^{\prime} u_{k}^{\prime} \otimes \alpha_{k}^{\prime} u_{k}^{\prime}}=\overline{\alpha^{\prime}{ }_{k}^{2} \cdot u_{k}^{\prime} \otimes u_{k}^{\prime}}$ and using Grüss-inequality once more

$$
\left|\overline{\alpha^{\prime}{ }_{k}^{2} \cdot u_{k}^{\prime} \otimes u_{k}^{\prime}}-\overline{\alpha_{k}^{\prime 2}} \cdot \overline{u_{k}^{\prime} \otimes u_{k}^{\prime}}\right| \leq \frac{\Delta_{\alpha_{k}^{\prime 2}} \cdot \Delta_{u_{k}^{\prime} \otimes u_{k}^{\prime}}}{4}
$$

Gathering various terms with regard to monotonies of variables $\alpha_{k}^{\prime 2}$ and $u_{k}^{\prime} \otimes u_{k}^{\prime}$, a bounded approximation of $\mathcal{R}_{\mathcal{F}}^{k}$ is obtained.

$$
\begin{aligned}
& \left|\mathcal{R}_{\mathcal{F}}^{k}-\overline{u_{k}^{\prime} \otimes u_{k}^{\prime}} \cdot\left(1+\sqrt{\frac{\overline{\alpha_{k}^{\prime 2}}}{\bar{\alpha}_{k}^{2}}}-\frac{\overline{\alpha_{k}^{\prime 2}}}{{\overline{\alpha_{k}}}^{2}}\right)\right| \leq \dot{\mathcal{R}}_{\mathcal{F}}^{k} \\
& \dot{\mathcal{R}}_{\mathcal{F}}^{k}=\frac{\Delta_{u_{k}^{\prime} \otimes u_{k}^{\prime}}}{2} \cdot\left(\sqrt{\frac{\overline{\alpha_{k}^{\prime 2}}}{{\overline{\alpha_{k}^{\prime}}}^{2}}}+\frac{\Delta_{\alpha_{k}^{\prime 2}}}{{\overline{\alpha_{k}^{\prime}}}^{2}}\right)
\end{aligned}
$$

Presuming that measurable fluctuations obey to non-skewed distributions, fluctuating primitive variables are thus such that $\psi_{\min }^{\prime} \approx-\psi_{\max }^{\prime}$. For second order statistics, $\Delta_{\psi^{\prime}} \approx \epsilon_{\psi^{\prime}} \ll 1$, thus $\lim _{\epsilon \rightarrow 0} \stackrel{\circ}{\mathcal{R}}_{\mathcal{F}}^{k}=0$. 


\section{Appendix}

Fluid mixture models which are reduced to a single material formulation (in the sense that the model is described by a single mixture pressure, velocity, and speed of sound) are practical because they are well-posed and relatively simple to implement. The mixture components occupy separate volumes at the same temperature and pressure. The continuity equation for the individual components $k$ using the total derivative form becomes:

$$
\rho_{k} \frac{d \alpha_{k}}{d t}+\alpha_{k} \frac{d \rho_{k}}{d t}+\rho_{k} \alpha_{k} \nabla \cdot \vec{u}=0
$$

Or, in terms of specific volumes, $\rho_{k}=\frac{1}{V_{k}}$ and $\nabla \vec{u}=\frac{1}{V} \frac{d V}{d t}$

$$
\frac{d \alpha_{k}}{d t}+\alpha_{k}\left(\frac{1}{V} \frac{d V}{d t}-\frac{1}{V_{k}} \frac{d V_{k}}{d t}\right)=0
$$

Single component volume fractions $\alpha_{k}$, mass fractions $\xi_{k}$, specific volumes $V_{k}$ are related to the mixture density $\rho$ and to the specific total volume $V$ by:

$$
\alpha_{k} \rho_{k}=\xi_{k} \rho \text { or } \alpha_{k} V=\xi_{k} V_{k} \text { with } V=\sum_{k=g, l} \xi_{k} V_{k}
$$

The thermodynamics quantities of mixture are pressure $p$, temperature $T$, specific entropy $S(p, V, \xi)$ and specific internal energy $U(p, V, \xi)$ are expressed as:

$$
S=\sum_{k=g, l} \xi_{k} S_{k}\left(p, V_{k}\right) \text { and } U(p, V, \xi)=\sum_{k=g, l} \xi_{k} U_{k}\left(p, V_{k}\right)
$$

The Gibbs identity for the mixture states:

$$
d U=T d S-p d V+\sum_{k=g, l} d \xi_{k}\left(\partial_{\xi_{k}} U_{k}\right)_{V, p}
$$

The absence of chemical reaction or phase change leads to vanishing chemical potentials; i.e $\left(\partial_{\xi_{k}} U_{k}\right)=0$. The extraction of entropy, pressure and density derivatives of a component $k$ from Gibbs relation along the trajectory of motion leads 
to:

$$
\begin{gathered}
T_{k} \frac{d S_{k}}{d t}=\left(\partial_{p} U_{k}\right)_{V} \frac{d p_{k}}{d t}+\left[\left(\partial_{V} U_{k}\right)_{p}+p_{k}\right] \frac{d V_{k}}{d t} \\
\frac{\beta T_{k}}{C_{p}} \frac{d S_{k}}{d t}=\frac{K_{T} C_{V}}{C_{p}} \frac{d p_{k}}{d t}+\frac{1}{V_{k}} \frac{d V_{k}}{d t}
\end{gathered}
$$

At this point, some fundamental quantities such as thermal expansion $\beta$, specific heat at constant pressure and temperature $C_{p}$ and $C_{V}$ respectively, were used:

$$
\begin{gathered}
\left(\partial_{T} V\right)_{p}=\beta V, \quad\left(\partial_{p} U\right)_{V}=\frac{K_{T} C_{V}}{\beta},\left(\partial_{V} U\right)_{p}=\frac{C_{p}}{\beta V}-p \\
T\left(\partial_{T} S\right)_{p, V}=C_{p, V} \quad, \quad \frac{K_{S}}{K_{T}}=\frac{C_{V}}{C_{p}}, \quad K_{S}=\frac{1}{\rho c^{2}}=\frac{1}{p \gamma}, \Gamma=\frac{\beta V}{K_{T} C_{V}}
\end{gathered}
$$

Involving isothermal and is-entropic compressibility factors, $K_{S}$ and $K_{T}$. The speed of sound $c$ and Grüneisen coefficient $\Gamma$ helped for the reformulation of the Gibbs identity:

$$
\frac{1}{V_{k}} \frac{d V_{k}}{d t}=\frac{1}{\gamma_{k}}\left(\Gamma_{k} \frac{T_{k}}{p_{k} V_{k}} \frac{d S_{k}}{d t}-\frac{1}{p_{k}} \frac{d p_{k}}{d t}\right)
$$

Considering that pressure and temperature are in equilibrium, i.e, $p_{k}=p, T_{k}=T$, the Gibbs relation in D.8 takes the form:

$$
\frac{1}{p} \frac{d p}{d t}=\frac{\gamma_{k}}{\rho_{k}} \frac{d \rho_{k}}{d t}+\frac{\Gamma_{k}}{\left(\frac{p V_{k}}{T}\right)} \frac{d S_{k}}{d t}
$$




\section{References}

[1] T. S. Yih, P. Griffith, Unsteady Momentum Fluxes in Two-phase Flow and the Vibration of Nuclear Reactor Components, Report No. AT(30-1)-3496.

[2] T. Yih, P. Griffith, Unsteady Momentum Fluxes in Two-phase Flow and the Vibration of Nuclear Reactor Components, Proceedings of the Int. Conf. on Flow-Induced Vibrations in Reactor system Components, Argone IL.

[3] B. Tay, R. Thorpe, Forces on pipe bend due to slug flow, Proceedings of the 3rd North American Multiphase Technology Conf. Banff, Canada (2002) $281-300$.

[4] B. Tay, R. Thorpe, Effects of Liquid Physical Properties on the Forces Acting on a Pipe Bend in GasLiquid Slug Flow, Chem. Eng. Research and Design 82 (3) (2004) 344-356. doi:10.1205/026387604322870453.

[5] B. Tay, R. Thorpe, Hydrodynamic forces acting on pipe bends in gasliquid slug flow, Chemical Eng. Research and Design 92 (2) (2014) 812-825. doi:10.1016/j.cherd.2013.08.012.

[6] L. Xing, H. Yeung, S. Lo, Investigation of slug flow induced forces on pipe bends applying STAR-OLGA coupling, Proceedings of the 15th Int. Conf. on Multiphase Production Technology, Cannes, France.

[7] A. Mack, J. Hrishikesh, S. Belfroid, Numerical Rebuilding of Dynamic Instabilities and Forces in Multiphase Pipe Bend Flow, Int. J. Comp. Meth. and Exp. Meas. 6 (2018) 358-372.

[8] J. L. Riverin, E. de Langre, M. Pettigrew, Fluctuating forces caused by internal two-phase flow on bends and tees, Journal of Sound and Vibration 298 (4-5) (2006) 1088-1098. doi:10.1016/J.JSV.2006.06.039. 
[9] C. Zhang, N. W. Mureithi, M. J. Pettigrew, Development of models correlating vibration excitation forces to dynamic characteristics of two-phase flow in a tube bundle, Int. Journal of Multiphase Flow 34 (2008) 1048-1057. doi:10.1016/j.ijmultiphaseflow.2008.05.001.

[10] Y. Liu, S. Miwa, T. Hibiki, M. Ishii, H. Morita, Y. Kondoh, K. Tanimoto, Experimental study of internal two-phase flow induced fluctuating force on a $90^{\circ}$ elbow, Chem. Eng. Science 76 (2012) 173-187. doi:10.1016/j.ces.2012.04.021.

[11] S. Miwa, T. Hibiki, M. Mori, Analysis of Flow-Induced Vibration Due to Stratified Wavy Two-Phase Flow, Journal of Fluids Eng. 138 (9). doi:10.1115/1.4033371.

[12] I. Hannoun, H. S. G. Fernando, E. J. List, Turbulence structure near sharp density interface, J. Fluid Mech. 189 (1988) 189-209.

[13] D. Lakehal, B. Smith, M. Milelli, Large Eddy Simulation of bubbly turbulent shear flows, Journal of turbulence 25 (2002) 1-21.

[14] M. Fulgosi, D. Lakehal, S. Banerjee, V. De Angelis, Direct numerical simulation of turbulence in a sheared air-water flow with a deformable interface, Journal of Fluid Mechanics 482 (2003) 319-345. doi:10.1017/S0022112003004154.

[15] J. McCaslin, O. Desjardins, Theoretical and computational modeling of turbulence / interface interactions, Center for Turbulence Research. Proceedings of the Summer Program (2014) 79-88.

[16] J. Hunt, T. Ishihara, D. Szubert, I. Asproulias, Y. Hoarau, M. Braza, Turbulence near interface-modelling and simulations, Advances in Fluid-Structure Interaction. Notes on Numerical Fluid Mechanics and Multidisciplinary Design 133 (2016) 283-292. 
[17] M. Gourma, P. G. Verdin, Two-phase slug flows in helical pipes: Slug frequency alterations and helicity fluctuations, Int. Journal of Multiphase Flow 86 (2016) 10-20. doi:10.1016/j.ijmultiphaseflow.2016.07.013.

[18] J. Hart, J. Ellenberger, P. J. Hamersma, Single and two-phase flow through helically coiled tubes, Chem. Eng. Science 43 (1986) 173-187.

[19] B. Nichols, C. Hirt, R. Hotchkiss, A fractional volume of fluid method for free boundary dynamics, 7th Int. Conf. on Numerical Methods in Fluid Dynamics. Lecture Notes in Physics 141 (1981) 304-309.

[20] K. Jongtae, M. Yadav, K. Seungjin, Characteristics of Secondary Flow Induced by 90-Degree Elbow in Turbulent Pipe Flow, Engineering Applications of Computational Fluid Mechanics 8 (2) (2014) 229-239. doi:10.1080/19942060.2014.11015509.

[21] Fluent, ANSYS Fluent 15.0 User's Guide, Fluent 15.0.

[22] V. Yakhot, S. Orszag, Renormalisation group analysis of turbulence, Journal of Scientific Computing 1 (1) (1986) 3-51.

[23] C. Weatheburn, Differential geometry of three dimensions, Cambridge University Press 1.

[24] R. Menikoff, B. Plohr, The riemann problem for fluid flow of real materials, Rev. Mod. Phys. 61 (1). doi:10.1115/1.4033371.

[25] Y. Yang, R. Zhang, Y. An, J. Wu, Steady vortex force theory and slenderwing flow diagnosis, Acta Mechanica Sinica 23 (6) (2007) 609-619.

[26] C. Marongiu, R. Tognaccini, M. Ueno, Lift and Lift-Induced Drag Computation by Lamb Vector Integration, AIAA Journal 51 (6) (2013) 1420-1430. doi:10.2514/1.J052104. 
[27] A. Tsinober, On one property of Lamb vector in isotropic turbulent flow, Physics of Fluids A: Fluid Dynamics 2 (4) (1990) 484-486.

[28] H. Marmanis, Analogy between the NavierStokes equations and Maxwell's equations: Application to turbulence, Physics of Fluids 10 (6) (1998) 14281437.

[29] G. Rousseaux, S. Seifer, V. Steinberg, A. Weibel, On the Lamb vector and the hydrodynamic charge, Experiments in Fluids 42 (2) (2006) 291-299.

[30] C. Bailly, C. Bogey, X. Gloerfelt, Some useful hybrid approaches for predicting aerodynamic noise, C. R. Mecanique 333 (333) (2005) 666-675. doi:10.1016/j.crme.2005.07.006.

[31] D. A. Donzis, S. Jagannathan, Fluctuations of thermodynamics variables in stationary compressible turbulence, Journal of Fluid Mechanics 733 (2013) 221-244. doi:10.1017/jfm2013.445.

[32] Y. Taitel, A. Dukler, A model for predicting flow regime transitions in horizontal and near horizontal gas-liquid flow, AIChE Journal 22 (1) (1976) $47-55$.

[33] I. G. Manolis, High Pressure Gas-Liquid Slug Flow, Ph.D. thesis, Department of Chemical Engineering and Chemical Technology, Imperial College of Science, Technology and Medecine (1995).

[34] D. J. Nicklin, J. O. Wilkes, J. Davidson, Two-phase flow in vertical tubes, Trans. Inst. Chem. Eng. 40 (1962) 61-68.

[35] H. Pineda-Preza, T. Kim, E. Pereyra, N. Ratkovich, CFD modeling of air and highly viscous liquid two-phase slug flow in horizontal pipes, Chem. Engng. Res. Design 136 (2018) 638-653. doi:10.1016/j.cherd.2018.06.023. 
[36] G. Erlebacher, M. Hussaini, C. Speziale, T. A. Zhang, Toward The LargeEddy Simulation of Compressible Turbulent Flows., ICASE Report 90 (1990) $1-43$.

[37] M. Matić, J. E. Pecarić, N. Ujević, On new estimation of the remainder in generalized Taylor's formula, Math. Ineq. and Appl. 2 (3) (1999) 343-361.

[38] S. S. Dragomir, A generalization of grüss inequality in inner product spaces and applications, J. Math. Anal. Appl. 237 (1999) 7482. 